\title{
The Protective Effect of Ellagic and Ferulic Acids against Oxidative Stress Induced by Gamma Radiation and Aluminium Chloride in Male Albino Rats
}

\author{
Ahmed M. Salem ${ }^{1}$, Tarek F. Mohammaden ${ }^{2}$, Mohamed A. M. Ali ${ }^{*}$, Enas A. Mohamed ${ }^{2}$ and Hesham \\ F. Hassan ${ }^{3}$ \\ ${ }^{1}$ Department of Biochemistry, Faculty of Science, Ain Shams University, 11566 Abbassia, Cairo, Egypt, \\ ${ }^{2}$ Nuclear Materials Authority, Cairo, Egypt, \\ ${ }^{3}$ Radiation Biology Department, National Center for Radiation Research and Technology, Atomic Energy Authority, \\ Cairo, Egypt
}

\section{A R T I C LE I N F O}

Article history:

Received 24 November 2015

Accepted 10 December 2015

Keywords:

Ellagic acid;

Ferulic acid;

$\gamma$ radiation;

Aluminium chloride;

Oxidative stress.

\begin{abstract}
A B S T R A C T
Ionizing radiation interacts with biological systems through the generation of free radicals, which induce oxidative stress leading to cellular damage and organ dysfunction. Aluminium ( $\mathrm{Al}$ ) and its salts are commonly used in daily life as it was believed that it was non-toxic. However, this element can negatively impact human health, at least in part, by direct interaction with enzymes known to protect the cells from oxidative damage. Naturally occurring antioxidants are being extensively analyzed for their ability to protect against oxidative stress. Ellagic acid (EA) and Ferulic acid (FA) are plant polyphenolic compounds, have gained attention due to their multiple biological activities. Herein, we investigated the potential protective effect of EA and FA against oxidative stress induced by $\gamma$ radiation and aluminium chloride $\left(\mathrm{AlCl}_{3}\right)$ in male albino rats. Rats were randomly divided into thirteen groups: a negative control group, 3 positive control groups ( $\gamma$-irradiated group, $\mathrm{AlCl}_{3}$-treated group and $\gamma$-irradiated $+\mathrm{AlCl}_{3 \text { - }}$ treated group) and 9 groups ( $3 \gamma$-irradiated groups, $3 \mathrm{AlCl}_{3}$-treated groups and 3 $\gamma$-irradiated $+\mathrm{AlCl}_{3}$-treated groups) treated with EA and/or FA. Serum liver enzyme activities as well as lipid profile were assessed. Levels of lipid peroxidation, protein oxidation and endogenous antioxidants as well as the concentrations of iron $(\mathrm{Fe})$ and zinc $(\mathrm{Zn})$ were estimated in the liver tissue homogenates. Furthermore, liver tissues were examined by histological analysis. EA and/or FA were found to alleviate lipid peroxidation and protein oxidation as well as improving the antioxidant status in $\gamma$-irradiated and/or $\mathrm{AlCl}_{3}$-treated groups. Oral administration of EA and FA, either alone or in combination, may be useful for ameliorating $\gamma$ radiation and/or $\mathrm{AlCl}_{3}$-induced oxidative damage.
\end{abstract}

\section{Introduction}

Oxidative stress represents a major issue in human health. It refers to the enhanced generation of reactive oxygen species (ROS) and/or depletion in the antioxidant defense system, causing an imbalance between prooxidants and antioxidants. Uncontrolled increases in oxidant concentrations can cause reactions in chain, mediated by free radicals, attacking proteins, lipids and DNA, affecting normal biological functions. Excessive production of ROS leads to altered enzymes activity, induction of DNA single- and double-strand

* Corresponding author.

E-mail address: m_aly_79@yahoo.com breaks, lipid peroxidation and protein oxidation. Many pathological conditions and illnesses are associated with either high levels of free radicals or with the reduction in free radical scavenging capacity ${ }^{[1]}$.

The steadily increasing applications of radiation in clinical practice, industrial and agricultural activities, on top of residual radio-activity resulting from nuclear test explosions, have a measurable impact contributing to possible radiation hazards in humans. The deleterious effects of ionizing radiation in biological systems are mainly mediated through the generation of ROS, which may contribute to radiation-induced cytotoxicity and to metabolic and morphologic changes in animals and 
humans. This indirect action plays an important role in the induction of oxidative stress leading to cellular damage and organ dysfunction ${ }^{[2]}$.

The primary sources of aluminium (Al) exposure are corn, yellow cheese, salt, herbs, spices, tea, cosmetics, cookware and containers. In addition, it is widely used in food additives and toothpaste. $\mathrm{Al}$ compounds are widely used in medicines, e.g., antacids, phosphate binders, buffered aspirins, vaccines and injectable allergens ${ }^{[3]}$. Environmental pollution with different Alcontaining compounds, especially industrial waste water, exposes people to higher than normal levels of $\mathrm{Al}$ [4]. Furthermore, particulate matter distributed by cement-producing factories contains a high amount of $\mathrm{Al}$ and populations residing near these facilities are exposed to this pollution ${ }^{[5]}$. Aluminium chloride $\left(\mathrm{AlCl}_{3}\right)$ can be found in food products and in drinking water derived from both natural sources and treatment methods ${ }^{[6]}$. Al is known to cause toxic effects to a variety of organ systems including brain, bone, kidney and blood. It has been suggested that there is a relationship between high level of $\mathrm{Al}$ and increased risk of a number of pathogenic disorders, such as microcytic anemia, osteomalacia and, possibly, neurodegenerative disorders ${ }^{[7]}$.

Naturally occurring antioxidants are being extensively analyzed for their ability to protect against oxidative stress and its deleterious consequences to human health. Phenolic acids are naturally occurring compounds that are widely distributed in fruits, vegetables, whole grains and beverages such as red wine and tea. They attract special attention because they exhibit a wide variety of health-protective properties such as free radical scavenging ${ }^{[8]}$.

Ellagic acid (EA) is a naturally occurring plant polyphenol. The highest levels of EA are found in numerous fruits and vegetables including blackberries, cranberries, pecans, pomegranates, raspberries, strawberries, walnuts, wolfberry, grapes, peach and other plant foods ${ }^{[9]}$. EA has antiproliferative and antioxidant properties in a number of in vitro and animal models. As with other polyphenol antioxidants, EA has a chemoprotective effect in cellular models by reducing oxidative stress. These properties have generated interest in potential human health benefits from the consumption of $\mathrm{EA}^{[\mathbf{1 0 ]}}$.

Ferulic acid (FA) is the most abundant natural phenolic compound in plant cell wall components. It is commonly found in fruits and vegetables such as tomatoes, sweet corn, and rice bran. Moreover, FA is found in the seeds of coffee, apple, artichoke, peanut and orange as well as in both seeds and cell walls of rice, wheat and oats plants. FA arises from metabolism of phenylalanine and tyrosine by the Shikimate pathway in plants. FA is an effective scavenger of free radicals and it has been described as a food additive to prevent lipid peroxidation ${ }^{[11]}$. FA can be characterized as a natural membrane antioxidant in humans and known to protect against cancer, cold, flu, influenza, skin aging and muscle wasting ${ }^{[12]}$.

In view of the importance of protecting mankind from the deleterious effects of $\gamma$ radiation and/or $\mathrm{AlCl}_{3}$ and in an attempt to understand the role and mechanism of the naturally occurring plant polyphenols as inhibitors of oxidative stress, the present study was designed to evaluate the potential protective effect of EA and/or FA against $\gamma$ radiation and/or $\mathrm{AlCl}_{3}$-induced oxidative stress.

\section{Materials and Methods}

\section{Experimental animals}

A total of 104 male albino rats of Sprague Dawley strain with body weight ranging from 100-110 $\mathrm{g}$ were obtained from the holding company for biological products and vaccines (VACSERA), Giza, Egypt. Animals were housed in polypropylene cages $(47 \times 34 \times 18 \mathrm{~cm})$ in an airconditioned room with $55 \%$ of humidity, controlled temperature $\left(25 \pm 2^{\circ} \mathrm{C}\right)$, and automatic lighting (alternating $12 \mathrm{~h}$ periods of light and dark) throughout the duration of the study. The animals were fed on a commercial standard pellet diet (PMI Nutrition, Shoreview, MN, USA) and fresh drinking water. The animals were allowed to acclimatize to the environmental conditions for one week before experiments. All animal handling procedures were approved by the ethics committee of the national center for radiation research and technology (NCRRT), Atomic Energy authority, Cairo, Egypt and in accordance with the recommendations for the proper care and use of laboratory animals (National Institutes of Health [NIH] publication No.85-23, revised 1996).

\section{Irradiation procedure}

Irradiation of animals was carried out at the NCRRT using a gamma cell-40 Cesium-137 irradiator (Best Theratronics Ltd., Ottawa, Ontario, Canada). Rats of irradiated groups were exposed to a single dose of gamma radiation $(6 \mathrm{~Gy}$; dose rate $=0.5 \mathrm{~Gy} /$ minute $){ }^{[13]}$.

\section{Chemicals}

$\mathrm{AlCl}_{3}$ was purchased from Merck KGaA (Darmstadt, Germany), while EA and FA were purchased from Sigma-Aldrich (St. Louis, MO, USA).

\section{Animal treatments}

$\mathrm{AlCl}_{3}(20 \mathrm{mg} / \mathrm{kg} \mathrm{bw}){ }^{[14]}$, EA $(60 \mathrm{mg} / \mathrm{kg} \mathrm{bw}){ }^{[10]}$ and FA $(20 \mathrm{mg} / \mathrm{kg} \mathrm{bw})^{[11]}$ dissolved in $0.2 \%$ dimethyl sulfoxide (DMSO) were administered once daily via intragastric tube for 8 weeks. It has been previously reported that the $\mathrm{LD}_{50}$ of $\mathrm{AlCl}_{3}$ when administrated orally to rats was 380 $\mathrm{mg} / \mathrm{kg}$ bw ${ }^{[14]}$. Rats of irradiated groups were exposed to gamma radiation on the day prior to overnight fasting at the end of the experimental period.

\section{Animal groups}

The experimental animals were randomly divided into 13 groups, categorized as a negative control group, 3 positive control groups, $3 \gamma$-irradiated groups, $3 \mathrm{AlCl}_{3}$ treated groups and $3 \mathrm{AlCl}_{3}$-treated $+\gamma$-irradiated groups. Each group containing eight rats $(n=8)$. The groups were treated as follows: 


\begin{tabular}{|c|c|c|}
\hline Group name & $\begin{array}{l}\text { Group } \\
\text { designation }\end{array}$ & Treatment \\
\hline \multicolumn{3}{|l|}{ I. Negative control group } \\
\hline Control & $\mathrm{C}$ & $1 \mathrm{~mL}$ of $0.2 \%$ DMSO \\
\hline \multicolumn{3}{|l|}{ II. Positive control groups } \\
\hline 1) $\gamma$-irradiated & $\gamma$ & A single dose of 6 Gy $\gamma$ radiation \\
\hline 2) $\mathrm{AlCl}_{3}$ & $\mathrm{AlCl}_{3}$ & $1 \mathrm{~mL}$ of $20 \mathrm{mg} / \mathrm{kg}$ bw $\mathrm{AlCl}_{3}$ \\
\hline 3) $\mathrm{AlCl}_{3}+\gamma$-irradiated & $\mathrm{AlCl}_{3}+\gamma$ & $\begin{array}{l}1 \mathrm{~mL} \text { of } 20 \mathrm{mg} / \mathrm{kg} \text { bw } \mathrm{AlCl}_{3}+\text { a single dose of } 6 \\
\text { Gy } \gamma \text { radiation }\end{array}$ \\
\hline \multicolumn{3}{|l|}{ III. $\gamma$-irradiated groups } \\
\hline 1) Ellagic acid $+\gamma$-irradiated & $\mathrm{EA}+\gamma$ & $\begin{array}{l}1 \mathrm{~mL} \text { of } 60 \mathrm{mg} / \mathrm{kg} \text { bw EA+ a single dose of } 6 \\
\text { Gy } \gamma \text { radiation }\end{array}$ \\
\hline 2) Ferulic acid $+\gamma$-irradiated & $\mathrm{FA}+\gamma$ & $\begin{array}{l}1 \mathrm{~mL} \text { of } 20 \mathrm{mg} / \mathrm{kg} \text { bw FA+ a single dose of } 6 \mathrm{~Gy} \\
\gamma \text { radiation }\end{array}$ \\
\hline $\begin{array}{l}\text { 3) Ellagic acid+Ferulic acid }+\gamma \text { - } \\
\text { irradiated }\end{array}$ & $\mathrm{EA}+\mathrm{FA}+\gamma$ & $\begin{array}{l}1 \mathrm{~mL} \text { of } 60 \mathrm{mg} / \mathrm{kg} \text { bw EA+1 } \mathrm{mL} \text { of } 20 \mathrm{mg} / \mathrm{kg} \\
\text { bw FA+ a single dose of } 6 \mathrm{~Gy} \gamma \text { radiation }\end{array}$ \\
\hline \multicolumn{3}{|l|}{ IV. $\mathrm{AlCl}_{3}$-treated groups } \\
\hline 1) Ellagic acid $+\mathrm{AlCl}_{3}$ & $\mathrm{EA}+\mathrm{AlCl}_{3}$ & $\begin{array}{l}1 \mathrm{~mL} \text { of } 60 \mathrm{mg} / \mathrm{kg} \text { bw EA+1 } \mathrm{mL} \text { of } 20 \mathrm{mg} / \mathrm{kg} \\
\mathrm{bw} \mathrm{AlCl}_{3}\end{array}$ \\
\hline 2) Ferulic acid+AICl & $\mathrm{FA}+\mathrm{AlCl}_{3}$ & $\begin{array}{l}1 \mathrm{~mL} \text { of } 20 \mathrm{mg} / \mathrm{kg} \text { bw FA+1 } \mathrm{mL} \text { of } 20 \mathrm{mg} / \mathrm{kg} \text { bw } \\
\mathrm{AlCl}_{3}\end{array}$ \\
\hline 3) Ellagic acid+Ferulic acid+ $+\mathrm{AlCl}_{3}$ & $\mathrm{EA}+\mathrm{FA}+\mathrm{AlCl}_{3}$ & $\begin{array}{l}1 \mathrm{~mL} \text { of } 60 \mathrm{mg} / \mathrm{kg} \text { bw EA+1 mL of } 20 \mathrm{mg} / \mathrm{kg} \\
\text { bw FA+1 mL of } 20 \mathrm{mg} / \mathrm{kg} \mathrm{bw} \mathrm{AlCl}_{3}\end{array}$ \\
\hline \multicolumn{3}{|l|}{ V. $\mathrm{AlCl}_{3}$-treated $+\gamma$-irradiated groups } \\
\hline 1) Ellagic acid $+\mathrm{AlCl}_{3}+\gamma$-irradiated & $\mathrm{EA}+\mathrm{AlCl}_{3}+\gamma$ & $\begin{array}{l}1 \mathrm{~mL} \text { of } 60 \mathrm{mg} / \mathrm{kg} \text { bw EA+1 } \mathrm{mL} \text { of } 20 \mathrm{mg} / \mathrm{kg} \\
\text { bw } \mathrm{AlCl}_{3}+\text { a single dose of } 6 \mathrm{~Gy} \gamma \text { radiation }\end{array}$ \\
\hline 2) Ferulic acid $+\mathrm{AICl}_{3}+\gamma$-irradiated & $\mathrm{FA}+\mathrm{AlCl}_{3}+\gamma$ & $\begin{array}{l}1 \mathrm{~mL} \text { of } 20 \mathrm{mg} / \mathrm{kg} \text { bw } \mathrm{FA}+1 \mathrm{~mL} \text { of } 20 \mathrm{mg} / \mathrm{kg} \mathrm{bw} \\
\mathrm{AlCl}_{3}+\text { a single dose of } 6 \mathrm{~Gy} \gamma \text { radiation }\end{array}$ \\
\hline $\begin{array}{l}\text { 3) Ellagic acid }+ \text { Ferulic acid }+\mathrm{AlCl}_{3}+\gamma- \\
\text { irradiated }\end{array}$ & $\mathrm{EA}+\mathrm{FA}+\mathrm{AlCl}_{3}+\gamma$ & $\begin{array}{l}1 \mathrm{~mL} \text { of } 60 \mathrm{mg} / \mathrm{kg} \mathrm{bw} \mathrm{EA}+1 \mathrm{~mL} \text { of } 20 \mathrm{mg} / \mathrm{kg} \\
\mathrm{bw} \mathrm{FA}+1 \mathrm{~mL} \text { of } 20 \mathrm{mg} / \mathrm{kg} \mathrm{bw} \mathrm{AlCl}_{3}+\text { a single } \\
\text { dose of } 6 \mathrm{~Gy} \gamma \text { radiation }\end{array}$ \\
\hline
\end{tabular}

\section{Blood samples collection}

At the end of the experimental period of 56 days, rats were kept fasting overnight and then anaesthetized by inhalation of diethyl ether. Blood samples were collected by cardiac puncture. The sera were separated by centrifugation at $3,000 \mathrm{~g}$ for 15 minutes and stored at $-80^{\circ} \mathrm{C}$ until analysis.

\section{Preparation of liver tissue homogenate}

The liver from dissected rats was immediately excised, blood was cleared off by several washings with ice cold phosphate buffered saline (PBS) and the tissues were weighed and immediately transferred to $-80^{\circ}$ freezer for storage till analysis. On the day of the analysis, the liver tissue samples were homogenized in $0.1 \mathrm{M}$ Tris- $\mathrm{HCl}$ buffer, pH 7.4 ( $2 \mathrm{ml} / 100 \mathrm{mg}$ tissue $)$. The homogenate was centrifuged at 3,000 rpm for 5 minutes and the supernatant was used for the estimation of biochemical parameters. The total proteins in the tissue extract were determined as previously described ${ }^{[15]}$.

\section{Biochemical investigations}

Serum levels of alanine aminotransferase (ALT), aspartate aminotransferase (AST) [16], alkaline phosphatase (ALP) ${ }^{[17]}$, gamma-glutamyltransferase (GGT) ${ }^{[18]}$, were determined using commercially available kits (Thermo Fisher Scientific Inc., Waltham, MA, USA) following the manufacturer's instructions. Total cholesterol (TC) ${ }^{[19]}$, high-density lipoprotein cholesterol (HDL-C) ${ }^{[20]}$ and triglycerides (TG) ${ }^{21]}$ serum levels were measured using commercially available kits (DiaSys Diagnostic Systems, Holzheim, Germany) according to the manufacturer's instructions. Lowdensity lipoprotein cholesterol (LDL-C) levels were calculated using the Friedewald formula ${ }^{[22]}$. Levels of malondialdehyde (MDA) ${ }^{[23]}$, protein carbonyl content (PCC) ${ }^{[24]}$ and reduced glutathione (GSH) ${ }^{[25]}$ as well as superoxide dismutase (SOD) activity ${ }^{[26]}$ were estimated in the liver tissue homogenates using $100 \mathrm{mg}$ of the liver tissue.

The colorimetric determination of MDA was based on the reaction of one molecule of MDA with two molecules of thiobarbituric acid (TBA) under high temperature $\left(90-100^{\circ} \mathrm{C}\right)$ and acidic conditions ( $\left.\mathrm{pH} 2-3\right)$ to form the pink-colored 1:2 MDA:TBA adduct, which was extracted by n-butanol, and its absorbance was measured at $535 \mathrm{~nm}$. The intensity of the pink color is directly proportional to the MDA content in the sample. PCC was determined by the derivatization of protein carbonyl groups with 2,4-dinitrophenylhydrazine 
(DNPH) leading to the formation of stable dinitrophenyl hydrazone adducts, which was detected spectrophotometrically at $375 \mathrm{~nm}$. The millimolar extinction coefficient of DNPH was used to calculate the carbonyl content.

An enzymatic recycling method, using glutathione reductase, was used for the quantification of GSH. The sulfhydryl group of GSH reacts with 5,5'-dithio-bis-2nitrobenzoic acid (DTNB; Ellman's reagent) and produces a yellow colored 5-thio-2-nitrobenzoic acid (TNB). The mixed disulfide between GSH and TNB that is concomitantly produced is reduced by glutathione reductase to recycle the GSH and produce more TNB. The rate of TNB production is directly proportional to this recycling reaction which is in turn directly proportional to the concentration of GSH in the sample. Accordingly, the measurement of the absorbance of TNB at $414 \mathrm{~nm}$ provides an accurate estimation of GSH in the sample.

SODs are metalloenzymes that catalyze the dismutation of the superoxide radical into $\mathrm{H}_{2} \mathrm{O}_{2}$ and $\mathrm{O}_{2}$. In the SOD assay, superoxide ions are generated from the conversion of xanthine and $\mathrm{O}_{2}$ to uric acid and $\mathrm{H}_{2} \mathrm{O}_{2}$ by xanthine oxidase. The superoxide anions then reduce a tetrazolium salt (nitroblue tetrazolium; NBT) to a colored formazan dye (NBT-diformazan), its absorbance was measured at $550 \mathrm{~nm}$. Addition of SOD to this reaction reduces superoxide ion levels, thereby lowering the rate of formazan dye formation. SOD activity in the sample is measured as the percent inhibition of the rate of formazan dye formation. One unit of SOD is defined as the amount of enzyme that inhibits the rate of NBT reduction by $50 \%$.

For the determination of $\mathrm{Fe}$ and $\mathrm{Zn}$ concentrations in the liver tissue homogenates, a microwave-assisted digestion of the tissues was performed. Briefly, approximately $100 \mathrm{mg}$ of the liver tissue was accurately weighed into a pre-cleaned microwave Teflon digestion vessel. Eight $\mathrm{ml}$ of nitric acid and $2 \mathrm{ml}$ of hydrogen peroxide were added into the reaction vessel and the digestion vessels were placed in the vessel rotor and into the high pressure microwave digestion system (MLS1200 Mega microwave oven, Milestone Srl, Sorisole, Italy) to completely convert the samples into an aqueous form following the manufacturer's instructions. Fe and $\mathrm{Zn}$ concentrations were determined using Perkin Elmer Model 23000 atomic absorption spectrophotometer ${ }^{\text {[27] }}$.

\section{Histological analysis}

The anterior portion of the left lateral lobe of the liver was sectioned and used for histological analysis. The tissue was fixed by immersion in $10 \%$ neutral-buffered formalin. The sample was then embedded in paraffin, sliced into 5- $\mu \mathrm{m}$ sections, stained with hematoxylineosin (H\&E), followed by blinded histological assessment. Histological changes were evaluated in nonconsecutive, randomly chosen $\times 400$ histological fields.

\section{Statistical analysis}

Data were expressed as mean \pm standard error of mean (SEM). The statistical significance of differences between means was analyzed using one-way analysis of variance (ANOVA) followed by Duncan's new multiple range test. Statistical significance was set at a $P$ value of $<0.05$. Data statistical analyses were performed using the statistical package for the social sciences (SPSS Statistics for Windows, Version 20.0; IBM Corp., Armonk, NY, USA).

\section{Results}

\section{Effect of EA and/or FA administration on hepatic} function

Serum AST, ALT, ALP and GGT activities in $\mathrm{AlCl}_{3^{-}}$ treated and/or $\gamma$-irradiated rats were significantly increased compared to those in the control rats. $\mathrm{AlCl}_{3}$ and/or $\gamma$-radiation-induced hepatic function impairment was alleviated by EA and/or FA administration as the activities of serum ALT, AST, ALP and GGT were markedly decreased (Table 1).

Effect of EA and/or FA administration on lipid profile

As shown in Table 2, $\mathrm{AlCl}_{3}$-treated and/or $\gamma$-irradiated rats had significantly higher serum TC, TG and LDL-C levels and lower HDL-C concentrations as compared to the control rats. Upon oral administration of EA and/or FA, $\mathrm{AlCl}_{3}$ and/or $\gamma$-radiation-induced dyslipidemia was ameliorated as serum TC, TG and LDL-C levels were significantly decreased and the HDL-C levels were markedly increased when compared to $\mathrm{AlCl}_{3}$-treated and/or $\gamma$-irradiated rats.

Effect of EA and/or FA administration on lipid peroxidation and protein oxidation levels in liver tissue

MDA and PCC levels were markedly elevated in liver tissue of $\mathrm{AlCl}_{3}$-treated and/or $\gamma$-irradiated rats compared to those in the control rats. Administration of EA and/or FA resulted in a significant reduction in MDA and PCC levels in comparison to $\mathrm{AlCl}_{3}$-treated and/or $\gamma$-irradiated rats (Table 3 ).

Effect of EA and/or FA administration on liver tissue antioxidant status

Table 4 shows that $\mathrm{AlCl}_{3}$ treatment and/or $\gamma$-radiation induced a remarkable depletion in liver tissue SOD activity and GSH content compared to the control rats, indicating a decline in antioxidant status. On the other hand, the antioxidant status was enhanced by EA and/or FA administration, which caused a significant elevation of SOD activity and GSH level in the liver tissue when compared to $\mathrm{AlCl}_{3}$-treated and/or $\gamma$-irradiated rats.

Effect of EA and/or FA administration on Fe and Zn concentrations in liver tissue

$\mathrm{AlCl}_{3}$-treated and/or $\gamma$-irradiated rats showed a marked elevation in $\mathrm{Fe}$ and $\mathrm{Zn}$ concentrations when compared to the rats in control the group. Compared to $\mathrm{AlCl}_{3}$-treated and/or $\gamma$-irradiated rats, $\mathrm{Fe}$ and $\mathrm{Zn}$ concentrations were significantly decreased upon EA and/or FA supplementation (Table 5).

Effect of EA and/or FA administration on hepatic tissue morphology

The histopathological analysis of hepatic sections from 
Table 1: Effects of EA and/or FA administration on serum liver enzymes activity in $\mathrm{AlCl}_{3}$-treated and/or $\gamma$-irradiated rats.

\begin{tabular}{|c|c|c|c|c|}
\hline Animal groups & $\begin{array}{l}\mathbf{A L T} \\
(\mathbf{U} / \mathbf{l}) \\
\end{array}$ & $\begin{array}{l}\text { AST } \\
(\mathbf{U} / \mathbf{l}) \\
\end{array}$ & $\begin{array}{l}\mathbf{A L P} \\
(\mathbf{U} / \mathbf{l}) \\
\end{array}$ & $\begin{array}{l}\text { GGT } \\
(\mathbf{U} / \mathbf{l}) \\
\end{array}$ \\
\hline $\mathrm{C}$ & $6.66 \pm 0.84$ & $10.66 \pm 0.42$ & $130.46 \pm 1.42$ & $2.21 \pm 0.11$ \\
\hline $\begin{array}{c}\gamma \\
\% \text { change } 1, P \text {-value }<\end{array}$ & $\begin{array}{c}18 \pm 1.0 \\
170.27,0.001 \\
\end{array}$ & $\begin{array}{l}19.66 \pm 0.66 \\
84.42,0.001\end{array}$ & $\begin{array}{c}194.76 \pm 0.67 \\
49.28,0.001 \\
\end{array}$ & $\begin{array}{c}3.96 \pm 0.29 \\
79.18,0.001 \\
\end{array}$ \\
\hline $\begin{array}{c}\mathrm{AlCl}_{3} \\
\% \text { change } 1, P \text {-value }<\end{array}$ & $\begin{array}{c}13.66 \pm 1.05 \\
105.10,0.001\end{array}$ & $\begin{array}{l}18.66 \pm 1.05 \\
75.04,0.001\end{array}$ & $\begin{array}{c}177.25 \pm 0.78 \\
35.86,0.001 \\
\end{array}$ & $\begin{array}{c}3.5 \pm 0.18 \\
58.37,0.001\end{array}$ \\
\hline $\begin{array}{c}\mathrm{AlCl}_{3}+\gamma \\
\% \text { change } 1, P \text {-value }<\end{array}$ & $\begin{array}{c}21 \pm 1.26 \\
215.31,0.001 \\
\end{array}$ & $\begin{array}{c}21.66 \pm 1.33 \\
103.18,0.001 \\
\end{array}$ & $\begin{array}{c}245.85 \pm 1.11 \\
88.44,0.001\end{array}$ & $\begin{array}{c}4.6 \pm 0.22 \\
108.14,0.001 \\
\end{array}$ \\
\hline $\begin{array}{c}\mathrm{EA}+\gamma \\
\% \text { change } 1, P \text {-value }< \\
\% \text { change } 2, P \text {-value }<\end{array}$ & $\begin{array}{c}10.6 \pm 0.8 \\
59.15,0.01 \\
-41.11,0.001 \\
\end{array}$ & $\begin{array}{c}16.5 \pm 0.92 \\
54.78,0.001 \\
-16.07,0.01 \\
\end{array}$ & $\begin{array}{r}169.06 \pm 0.73 \\
29.58,0.001 \\
-13.19,0.001 \\
\end{array}$ & $\begin{array}{c}2.98 \pm 0.11 \\
34.84,0.01 \\
-24.74,0.001 \\
\end{array}$ \\
\hline $\begin{array}{c}\text { FA+ } \gamma \\
\% \text { change } 1, P \text {-value }< \\
\% \text { change } 2, P \text {-value }<\end{array}$ & $\begin{array}{c}10.6 \pm 0.8 \\
59.15,0.01 \\
-41.11,0.001 \\
\end{array}$ & $\begin{array}{c}15.5 \pm 0.92 \\
45.4,0.001 \\
-21.15,0.001 \\
\end{array}$ & $\begin{array}{r}167.68 \pm 0.52 \\
28.52,0.001 \\
-13.9,0.001 \\
\end{array}$ & $\begin{array}{c}2.95 \pm 0.11 \\
33.48,0.01 \\
-25.5,0.001 \\
\end{array}$ \\
\hline $\begin{array}{c}\text { EA+FA+ } \gamma \\
\% \text { change } 1, P \text {-value }< \\
\% \text { change } 2, P \text {-value }<\end{array}$ & $\begin{array}{c}8.6 \pm 0.42 \\
29.19, \text { N.S } \\
-52.22,0.001 \\
\end{array}$ & $\begin{array}{c}14.5 \pm 0.89 \\
36.02,0.001 \\
-26.24,0.001 \\
\end{array}$ & $\begin{array}{c}148.25 \pm 0.7 \\
13.63,0.001 \\
-23.88,0.001 \\
\end{array}$ & $\begin{array}{c}2.48 \pm 0.17 \\
12.21, \mathrm{~N} . \mathrm{S} \\
-37.37,0.001 \\
\end{array}$ \\
\hline $\begin{array}{c}\mathrm{EA}+\mathrm{AlCl}_{3} \\
\% \text { change } 1, P \text {-value }< \\
\% \text { change } 3, P \text {-value }<\end{array}$ & $\begin{array}{c}10.16 \pm 0.65 \\
52.55,0.01 \\
-25.62,0.01 \\
\end{array}$ & $\begin{array}{c}14.16 \pm 0.54 \\
32.83,0.01 \\
-24.11,0.001 \\
\end{array}$ & $\begin{array}{c}154.9 \pm 0.87 \\
18.73,0.001 \\
-12.6,0.001 \\
\end{array}$ & $\begin{array}{c}2.71 \pm 0.12 \\
22.62,0.05 \\
-22.57,0.01 \\
\end{array}$ \\
\hline $\begin{array}{c}\mathrm{FA}^{2} \mathrm{AlCl}_{3} \\
\% \text { change } 1, P \text {-value }< \\
\% \text { change } 3, P \text {-value }<\end{array}$ & $\begin{array}{c}9.8 \pm 0.74 \\
47.14,0.05 \\
-28.25,0.01 \\
\end{array}$ & $\begin{array}{c}14.4 \pm 0.55 \\
35.08,0.001 \\
-22.82,0.001 \\
\end{array}$ & $\begin{array}{c}155.54 \pm 0.78 \\
19.22,0.001 \\
-12.24,0.001 \\
\end{array}$ & $\begin{array}{c}2.71 \pm 0.12 \\
22.62,0.05 \\
-22.57,0.01 \\
\end{array}$ \\
\hline $\begin{array}{c}\text { EA+FA+AlCl } \\
\% \text { change } 1, P \text {-value }< \\
\% \text { change } 3, P \text {-value }<\end{array}$ & $\begin{array}{c}7.3 \pm 0.66 \\
9.6, \text { N.S } \\
-46.55,0.001 \\
\end{array}$ & $\begin{array}{c}13.9 \pm 0.49 \\
30.39,0.01 \\
-25.5,0.001 \\
\end{array}$ & $\begin{array}{c}144.09 \pm 0.97 \\
10.44,0.001 \\
-18.7,0.001 \\
\end{array}$ & $\begin{array}{c}2.5 \pm 0.14 \\
13.12, \text { N.S } \\
-28.57,0.001 \\
\end{array}$ \\
\hline $\begin{array}{c}\mathrm{EA}+\mathrm{AlCl}_{3}+\gamma \\
\% \text { change } 1, P \text {-value }< \\
\% \text { change } 4, P \text {-value }<\end{array}$ & $\begin{array}{r}12.16 \pm 1.16 \\
82.58,0.001 \\
-42.09,0.001 \\
\end{array}$ & $\begin{array}{l}18.8 \pm 0.16 \\
76.36,0.001 \\
-13.2,0.05 \\
\end{array}$ & $\begin{array}{c}218.83 \pm 0.44 \\
67.73,0.001 \\
-10.99,0.001 \\
\end{array}$ & $\begin{array}{c}3.1 \pm 0.26 \\
40.27,0.001 \\
-32.6,0.001 \\
\end{array}$ \\
\hline $\begin{array}{c}\mathrm{FA}+\mathrm{AlCl}_{3}+\gamma \\
\% \text { change } 1, P \text {-value }< \\
\% \text { change } 4, P \text {-value }<\end{array}$ & $\begin{array}{r}11.33 \pm 0.66 \\
70.12,0.001 \\
-46.04,0.001 \\
\end{array}$ & $\begin{array}{c}17.5 \pm 0.67 \\
64.16,0.001 \\
-19.2,0.001 \\
\end{array}$ & $\begin{array}{l}222.43 \pm 0.78 \\
70.49,0.001 \\
-9.52,0.001 \\
\end{array}$ & $\begin{array}{c}3.2 \pm 0.14 \\
44.79,0.001 \\
-30.43,0.001 \\
\end{array}$ \\
\hline $\begin{array}{c}\text { EA+FA+AlCl }+\gamma \\
\% \text { change } 1, P \text {-value }< \\
\% \text { change } 4, P \text {-value }<\end{array}$ & $\begin{array}{c}9.33 \pm 0.84 \\
40.09,0.05 \\
-55.57,0.001 \\
\end{array}$ & $\begin{array}{c}14.5 \pm 0.67 \\
36.02,0.001 \\
-33.05,0.001 \\
\end{array}$ & $\begin{array}{l}184.86 \pm 0.88 \\
41.69,0.001 \\
-24.8,0.001 \\
\end{array}$ & $\begin{array}{c}2.68 \pm 0.11 \\
21.26, \text { N.S } \\
-41.73,0.001 \\
\end{array}$ \\
\hline
\end{tabular}

ALT, alanine aminotransferase; AST, aspartate aminotransferase; ALP, alkaline phosphatase; GGT, gamma-glutamyltransferase; C, Control; $\gamma, \gamma$ irradiated; $\mathrm{AlCl}_{3}, \mathrm{AlCl}_{3}$-treated; $\mathrm{AlCl}_{3}+\gamma, \mathrm{AlCl}_{3}$-treated $+\gamma$-irradiated; $\mathrm{EA}+\gamma$, Ellagic acid $+\gamma$-irradiated; $\mathrm{FA}+\gamma$, Ferulic acid $+\gamma$-irradiated; $\mathrm{EA}+\mathrm{FA}+\gamma$, Ellagic acid + Ferulic acid $+\gamma$-irradiated; $\mathrm{EA}+\mathrm{AlCl}_{3}$, Ellagic acid $+\mathrm{AlCl}_{3}$-treated; $\mathrm{FA}+\mathrm{AlCl}_{3}$, Ferulic acid $+\mathrm{AlCl}_{3}$-treated; $\mathrm{EA}+\mathrm{FA}+\mathrm{AlCl}_{3}$, Ellagic acid + Ferulic acid $+\mathrm{AlCl}_{3}$-treated; $\mathrm{EA}+\mathrm{AlCl}_{3}+\gamma$, Ellagic acid $+\mathrm{AlCl}_{3}$-treated $+\gamma$-irradiated; $\mathrm{FA}+\mathrm{AlCl}_{3}+\gamma, \mathrm{Ferulic}$ acid + $\mathrm{AlCl}_{3}$-treated $+\gamma$-irradiated; $\mathrm{EA}+\mathrm{FA}+\mathrm{AlCl}_{3}+\gamma$, Ellagic acid + Ferulic acid $+\mathrm{AlCl}_{3}$-treated $+\gamma$-irradiated; N.S: non-significant $(P>0.05)$. Data are represented as mean $\pm \operatorname{SEM}(\mathrm{n}=8) . \%$ change 1 , compared to control group; $\%$ change 2 , compared to $\gamma$-irradiated group; $\%$ change 3 , compared to $\mathrm{AlCl}_{3}$-treated group; \% change 4 , compared to $\mathrm{AlCl}_{3}$-treated $+\gamma$-irradiated group.

control rats revealed normal liver histological architecture (Figure 1A). In contrast, hepatocytes cytoplasmic vacuolization, focal hepatic hemorrhage, congestion of hepatoportal blood vessel and deposition of collagen fibers in portal tract were observed in hepatic sections from $\mathrm{AlCl}_{3}$-treated and/or $\gamma$-irradiated rats (Figure 1B-1D). These histological alterations were somewhat attenuated by oral administration of EA and/or FA (Figure 1E-1M).

\section{Discussion}

Exposure to ionizing radiation represents a genuine, increasing threat to mankind and our environment. The exposure to ionizing radiation yields ROS, which causes oxidative degradation of all types of cellular biomolecules and reduces the activity of antioxidant enzymes ${ }^{[28]}$. $\mathrm{Al}$ is found in combination with oxygen, silicon, fluorine and other elements in the soil, rocks, clays and gems. The use of such tools may increase an individual's Al exposure, particularly when these are used with salty, acidic or alkaline foods ${ }^{[29]}$. It has also been suggested that the toxic effects associated with $\mathrm{Al}$ are due to the generation of ROS, which results in the oxidative deterioration of cellular lipids, proteins and DNA ${ }^{[30]}$. However, humans have evolved highly 
Table 2: Effects of EA and/or FA administration on serum lipid profile in $\mathrm{AlCl}_{3}$-treated and/or $\gamma$-irradiated rats.

\begin{tabular}{|c|c|c|c|c|}
\hline Animal groups & $\begin{array}{c}\mathrm{TC} \\
(\mathrm{mg} / \mathrm{dl})\end{array}$ & $\begin{array}{c}\text { TG } \\
(\mathrm{mg} / \mathrm{dl})\end{array}$ & $\begin{array}{l}\text { HDL-C } \\
\text { (mg/dl) }\end{array}$ & $\begin{array}{l}\text { LDL-C } \\
\text { (mg/dl) }\end{array}$ \\
\hline C & $65.10 \pm 2.34$ & $48.75 \pm 2.33$ & $47.88 \pm 2.39$ & $11.91 \pm 0.97$ \\
\hline $\begin{array}{c}\gamma \\
\% \text { change } 1, P \text {-value }<\end{array}$ & $\begin{array}{c}78.5 \pm 0.84 \\
20.58,0.001\end{array}$ & $\begin{array}{l}70.04 \pm 1.34 \\
43.67,0.001\end{array}$ & $\begin{array}{c}26.21 \pm 0.29 \\
-45.25,0.001\end{array}$ & $\begin{array}{c}38.27 \pm 0.84 \\
221.32,0.001\end{array}$ \\
\hline $\begin{array}{c}\mathrm{AlCl}_{3} \\
\% \text { change } 1, P \text {-value }<\end{array}$ & $\begin{array}{c}70.16 \pm 0.47 \\
7.77,0.01\end{array}$ & $\begin{array}{l}59.16 \pm 0.74 \\
21.35,0.001\end{array}$ & $\begin{array}{c}29.32 \pm 0.31 \\
-38.76,0.001\end{array}$ & $\begin{array}{c}29.01 \pm 0.72 \\
143.57,0.001\end{array}$ \\
\hline $\begin{array}{c}\mathrm{AlCl}_{3}+\gamma \\
\% \text { change } 1, P \text {-value }<\end{array}$ & $\begin{array}{l}94.33 \pm 1.05 \\
44.90,0.001\end{array}$ & $\begin{array}{c}112 \pm 3.5 \\
129.74,0.001\end{array}$ & $\begin{array}{c}23.99 \pm 0.94 \\
-49.89,0.001\end{array}$ & $\begin{array}{c}48.5 \pm 1.6 \\
307.22,0.001\end{array}$ \\
\hline $\begin{array}{c}\text { EA }+\gamma \\
\% \text { change } 1, P \text {-value }< \\
\% \text { change } 2, P \text {-value }<\end{array}$ & $\begin{array}{c}73.16 \pm 0.74 \\
12.38,0.001 \\
-6.8,0.01 \\
\end{array}$ & $\begin{array}{c}67.16 \pm 0.6 \\
37.76,0.001 \\
-4.11, \text { N.S } \\
\end{array}$ & $\begin{array}{c}35.83 \pm 1.3 \\
-25.16,0.001 \\
36.7,0.001 \\
\end{array}$ & $\begin{array}{c}23.9 \pm 1.7 \\
100.67,0.001 \\
-37.54,0.001 \\
\end{array}$ \\
\hline $\begin{array}{c}\text { FA }+\gamma \\
\% \text { change } 1, P \text {-value }< \\
\% \text { change } 2, P \text {-value }<\end{array}$ & $\begin{array}{c}71.33 \pm 0.61 \\
9.56,0.001 \\
-9.13,0.001\end{array}$ & $\begin{array}{c}64.16 \pm 0.54 \\
31.61,0.001 \\
-8.39,0.05\end{array}$ & $\begin{array}{l}35.33 \pm 0.61 \\
-26.21,0.001 \\
34.79,0.001 \\
\end{array}$ & $\begin{array}{c}23.16 \pm 1.2 \\
94.45,0.001 \\
-39.48,0.001\end{array}$ \\
\hline $\begin{array}{c}\mathrm{EA}+\mathrm{FA}+\gamma \\
\% \text { change } 1, P \text {-value }< \\
\% \text { change } 2, P \text {-value }<\end{array}$ & $\begin{array}{c}66.5 \pm 0.92 \\
2.15, \text { N.S } \\
-15.28,0.001 \\
\end{array}$ & $\begin{array}{c}53.33 \pm 0.61 \\
9.39, \text { N.S } \\
-23.85,0.001 \\
\end{array}$ & $\begin{array}{l}39.66 \pm 0.76 \\
-17.16,0.001 \\
51.31,0.001 \\
\end{array}$ & $\begin{array}{c}16.16 \pm 0.78 \\
35.68,0.05 \\
-57.77,0.001\end{array}$ \\
\hline $\begin{array}{c}\text { EA+ }+\mathrm{AlCl}_{3} \\
\% \text { change } 1, P \text {-value }< \\
\% \text { change } 3, P \text {-value }<\end{array}$ & $\begin{array}{c}67.5 \pm 0.56 \\
3.68, \text { N.S } \\
-3.79, \text { N.S }\end{array}$ & $\begin{array}{c}51 \pm 0.68 \\
4.61, \text { N.S } \\
-13.79,0.01\end{array}$ & $\begin{array}{l}38.83 \pm 0.3 \\
-18.9,0.001 \\
32.43,0.001\end{array}$ & $\begin{array}{c}18.46 \pm 0.83 \\
54.99,0.01 \\
-36.36,0.001\end{array}$ \\
\hline $\begin{array}{c}\mathrm{FA}+\mathrm{AlCl}_{3} \\
\% \text { change } 1, P \text {-value }< \\
\% \text { change } 3, P \text {-value }<\end{array}$ & $\begin{array}{r}68 \pm 0.36 \\
4.45, \text { N.S } \\
-3.07, \text { N.S }\end{array}$ & $\begin{array}{c}50.66 \pm 0.55 \\
3.91, \text { N.S } \\
-14.36,0.01\end{array}$ & $\begin{array}{c}40.5 \pm 0.92 \\
-15.41,0.001 \\
38.13,0.001 \\
\end{array}$ & $\begin{array}{c}17.36 \pm 0.84 \\
45.75,0.01 \\
-40.15,0.001 \\
\end{array}$ \\
\hline $\begin{array}{c}\text { EA+FA+AlCl } \\
\% \text { change } 1, P \text {-value }< \\
\% \text { change } 3, P \text {-value }<\end{array}$ & $\begin{array}{c}67.5 \pm 0.61 \\
3.68, \text { N.S } \\
-3.79, \text { N.S } \\
\end{array}$ & $\begin{array}{c}50.66 \pm 0.55 \\
3.91, \text { N.S } \\
-14.36,0.01 \\
\end{array}$ & $\begin{array}{l}40.66 \pm 0.76 \\
-15.07,0.001 \\
38.67,0.001 \\
\end{array}$ & $\begin{array}{c}16.7 \pm 1.2 \\
40.21,0.05 \\
-42.43,0.001 \\
\end{array}$ \\
\hline $\begin{array}{c}\text { EA+AlCl}+\gamma \\
\% \text { change } 1, P \text {-value }< \\
\% \text { change } 4, P \text {-value }<\end{array}$ & $\begin{array}{c}86.33 \pm 1.14 \\
32.61,0.001 \\
-8.48,0.001\end{array}$ & $\begin{array}{c}82.58 \pm 2.2 \\
69.39,0.001 \\
-26.26,0.001\end{array}$ & $\begin{array}{c}28.91 \pm 0.77 \\
-39.61,0.001 \\
20.5,0.001\end{array}$ & $\begin{array}{c}40.88 \pm 1.6 \\
243.24,0.001 \\
-15.71,0.001\end{array}$ \\
\hline $\begin{array}{c}\mathrm{FA}+\mathrm{AlCl}_{3}+\gamma \\
\% \text { change } 1, P \text {-value }< \\
\% \text { change } 4, P \text {-value }<\end{array}$ & $\begin{array}{c}78.50 \pm 2.04 \\
20.58,0.001 \\
-16.78,0.001\end{array}$ & $\begin{array}{c}78.25 \pm 4.63 \\
60.51,0.001 \\
-30.13,0.001\end{array}$ & $\begin{array}{c}31.03 \pm 0.47 \\
-35.19,0.001 \\
29.34,0.001 \\
\end{array}$ & $\begin{array}{c}31.81 \pm 1.9 \\
167.08,0.001 \\
-34.41,0.001\end{array}$ \\
\hline $\begin{array}{c}\text { EA+FA+AlCl}+\gamma \\
\% \text { change } 1, P \text {-value }< \\
\% \text { change } 4, P \text {-value }<\end{array}$ & $\begin{array}{c}69.16 \pm 2.23 \\
6.23,0.05 \\
-26.68,0.001 \\
\end{array}$ & $\begin{array}{c}66.91 \pm 1.38 \\
37.25,0.001 \\
-40.25,0.001 \\
\end{array}$ & $\begin{array}{c}38.33 \pm 0.42 \\
-19.94,0.001 \\
59.77,0.001 \\
\end{array}$ & $\begin{array}{c}17.28 \pm 2.5 \\
45.08,0.01 \\
-64.37,0.001 \\
\end{array}$ \\
\hline
\end{tabular}

TC, total cholesterol; TG, triglycerides; HDL-C, high-density lipoprotein cholesterol; LDL-C, low-density lipoprotein cholesterol; C, Control; $\gamma$, $\gamma$-irradiated; $\mathrm{AlCl}_{3}, \mathrm{AlCl}_{3}$-treated; $\mathrm{AlCl}_{3}+\gamma, \mathrm{AlCl}_{3}$-treated $+\gamma$-irradiated; EA $+\gamma$, Ellagic acid $+\gamma$-irradiated; $\mathrm{FA}+\gamma$, Ferulic acid $+\gamma$-irradiated; $\mathrm{EA}+\mathrm{FA}+\gamma$, Ellagic acid + Ferulic acid $+\gamma$-irradiated; EA+AlCl 3 , Ellagic acid $+\mathrm{AlCl}_{3}$-treated; $\mathrm{FA}+\mathrm{AlCl}_{3}$, Ferulic acid $+\mathrm{AlCl}_{3}$-treated; $\mathrm{EA}+\mathrm{FA}+\mathrm{AlCl}_{3}$, Ellagic acid + Ferulic acid $+\mathrm{AlCl}_{3}$-treated; $\mathrm{EA}+\mathrm{AlCl}_{3}+\gamma$, Ellagic acid $+\mathrm{AlCl}_{3}$-treated $+\gamma$-irradiated; $\mathrm{FA}+\mathrm{AlCl}_{3}+\gamma, \mathrm{Ferulic}$ acid + $\mathrm{AlCl}_{3}$-treated $+\gamma$-irradiated; $\mathrm{EA}+\mathrm{FA}+\mathrm{AlCl}_{3}+\gamma$, Ellagic acid + Ferulic acid $+\mathrm{AlCl}_{3}$-treated $+\gamma$-irradiated; N.S: non-significant $(P>0.05)$. Data are represented as mean $\pm \operatorname{SEM}(\mathrm{n}=8)$. \% change 1, compared to control group; \% change 2, compared to $\gamma$-irradiated group; \% change 3 , compared to $\mathrm{AlCl}_{3}$-treated group; \% change 4, compared to $\mathrm{AlCl}_{3}$-treated $+\gamma$-irradiated group.

sophisticated endogenous antioxidant systems which play a crucial role in scavenging ROS and reducing oxidative damage.

Oxidative stress is an imbalance between the production of ROS and the antioxidant capacity. The ROS-mediated cell injury could be partially prevented by antioxidants and free radical scavengers. Plant-derived polyphenolic compounds including EA and FA have attracted considerable attention for their antioxidant properties. These dietary polyphenols are known to scavenge ROS and inhibit the oxidative damage induced by ROS, thereby reducing the risk of oxidative stress. There is a growing interest in understanding the role and mechanism of these compounds as inhibitors of oxidative stress. The present study aimed to evaluate the protective effect of EA and FA, either alone or in combination against oxidative damage induced by $\gamma$ radiation and/or $\mathrm{AlCl}_{3}$.

In our study, increased ALT, AST, ALP and GGT activities in $\gamma$-irradiated rats could be interpreted as a consequence of the radiation-induced free radical production, which attack the highly unsaturated fatty acids of the cell membrane to induce lipid peroxidation which is considered a key process in many pathological events and is one of the reactions induced by oxidative stress ${ }^{[31]}$. In the same regard, $\mathrm{AlCl}_{3}$-treated rats showed 
Table 3: Effects of EA and/or FA administration on liver tissue MDA and PCC levels in $\mathrm{AlCl}_{3}$-treated and/or $\gamma$ irradiated rats.

\begin{tabular}{|c|c|c|}
\hline Animal groups & $\begin{array}{c}\text { MDA } \\
\text { (nmol/g tissue) }\end{array}$ & $\begin{array}{c}\text { PCC } \\
\text { (nmol/mg protein) } \\
\end{array}$ \\
\hline $\mathbf{C}$ & $723.48 \pm 25.91$ & $13.73 \pm 0.25$ \\
\hline $\begin{array}{c}\gamma \\
\% \text { change } 1, P \text {-value }<\end{array}$ & $\begin{array}{c}1109.21 \pm 26.26 \\
53.31,0.001\end{array}$ & $\begin{array}{l}35.40 \pm 0.25 \\
157.82,0.001\end{array}$ \\
\hline $\begin{array}{c}\mathrm{AlCl}_{3} \\
\% \text { change } 1, P \text {-value }<\end{array}$ & $\begin{array}{c}1075.77 \pm 7.55 \\
48.69,0.001\end{array}$ & $\begin{array}{c}29.20 \pm 0.73 \\
112.67,0.001\end{array}$ \\
\hline $\begin{array}{c}\mathrm{AlCl}_{3}+\gamma \\
\% \text { change } 1, P \text {-value }<\end{array}$ & $\begin{array}{c}1456.51 \pm 24.74 \\
101.32,0.001 \\
\end{array}$ & $\begin{array}{l}44.28 \pm 0.23 \\
222.5,0.001 \\
\end{array}$ \\
\hline $\begin{array}{c}\text { EA }+\gamma \\
\% \text { change } 1, P \text {-value }< \\
\% \text { change } 2, P \text {-value }< \\
\end{array}$ & $\begin{array}{r}887.95 \pm 2.3 \\
22.73,0.001 \\
-19.94,0.001 \\
\end{array}$ & $\begin{array}{c}20.1 \pm 0.61 \\
46.39,0.001 \\
-43.22,0.001 \\
\end{array}$ \\
\hline $\begin{array}{c}\text { FA }+\gamma \\
\% \text { change } 1, P \text {-value }< \\
\% \text { change } 2, P \text {-value }<\end{array}$ & $\begin{array}{r}887.43 \pm 3.9 \\
22.66,0.001 \\
-19.99,0.001 \\
\end{array}$ & $\begin{array}{c}22.7 \pm 0.46 \\
65.33,0.001 \\
-35.87,0.001 \\
\end{array}$ \\
\hline $\begin{array}{c}\mathrm{EA}+\mathrm{FA}+\gamma \\
\% \text { change } 1, P \text {-value }< \\
\% \text { change } 2, P \text {-value }< \\
\end{array}$ & $\begin{array}{c}776.16 \pm 2 \\
7.28,0.01 \\
-30.02,0.001 \\
\end{array}$ & $\begin{array}{c}17.4 \pm 0.26 \\
26.72,0.001 \\
-50.84,0.001 \\
\end{array}$ \\
\hline $\begin{array}{c}\text { EA+AlCl } \mathrm{Al}_{3} \\
\% \text { change } 1, P \text {-value }< \\
\% \text { change } 3, P \text {-value }< \\
\end{array}$ & $\begin{array}{c}879.79 \pm 0.82 \\
21.6,0.001 \\
-18.21,0.001 \\
\end{array}$ & $\begin{array}{c}17.2 \pm 0.29 \\
25.27,0.001 \\
-41.09,0.001 \\
\end{array}$ \\
\hline $\begin{array}{c}\text { FA+AlCl } 3 \\
\% \text { change } 1, P \text {-value }< \\
\% \text { change } 3, P \text {-value }<\end{array}$ & $\begin{array}{r}872.71 \pm 1.3 \\
20.62,0.001 \\
-18.87,0.001 \\
\end{array}$ & $\begin{array}{c}18.9 \pm 0.37 \\
37.65,0.001 \\
-35.27,0.001 \\
\end{array}$ \\
\hline $\begin{array}{c}\text { EA+FA+AlCl } \\
\% \text { change } 1, P \text {-value }< \\
\% \text { change } 3, P \text {-value }<\end{array}$ & $\begin{array}{c}763.3 \pm 2.2 \\
5.5,0.05 \\
-29.04,0.001 \\
\end{array}$ & $\begin{array}{c}16.7 \pm 0.28 \\
21.63,0.001 \\
-42.8,0.001 \\
\end{array}$ \\
\hline $\begin{array}{c}\mathrm{EA}+\mathrm{AlCl}_{3}+\gamma \\
\% \text { change } 1, P \text {-value }< \\
\% \text { change } 4, P \text {-value }<\end{array}$ & $\begin{array}{r}912.56 \pm 0.42 \\
26.13,0.001 \\
-37.34,0.001 \\
\end{array}$ & $\begin{array}{r}31.25 \pm 0.68 \\
127.6,0.001 \\
-29.42,0.001 \\
\end{array}$ \\
\hline $\begin{array}{c}\mathrm{FA}+\mathrm{AlCl}_{3}+\gamma \\
\% \text { change } 1, P \text {-value }< \\
\% \text { change } 4, P \text {-value }< \\
\end{array}$ & $\begin{array}{c}906.57 \pm 2.43 \\
25.3,0.001 \\
-37.75,0.001 \\
\end{array}$ & $\begin{array}{c}29.05 \pm 0.57 \\
111.58,0.001 \\
-34.39,0.001 \\
\end{array}$ \\
\hline $\begin{array}{c}\text { EA+FA+AlCl }+\gamma \\
\% \text { change } 1, P \text {-value }< \\
\% \text { change } 4, P \text {-value }< \\
\end{array}$ & $\begin{array}{c}829.60 \pm 0.92 \\
14.66,0.001 \\
-43.04,0.001 \\
\end{array}$ & $\begin{array}{c}22.93 \pm 0.43 \\
67,0.001 \\
-48.21,0.001 \\
\end{array}$ \\
\hline
\end{tabular}

MDA, malondialdehyde; PCC, protein carbonyl content; $\mathrm{C}$, Control; $\gamma, \gamma$-irradiated; $\mathrm{AlCl}_{3}, \mathrm{AlCl}_{3}$-treated; $\mathrm{AlCl}_{3}+\gamma, \mathrm{AlCl}_{3}$-treated $+\gamma$-irradiated; EA $+\gamma$, Ellagic acid $+\gamma$-irradiated; FA $+\gamma$, Ferulic acid $+\gamma$-irradiated; EA+FA $+\gamma$, Ellagic acid + Ferulic acid $+\gamma$-irradiated; EA $+\mathrm{AlCl}_{3}$, Ellagic acid $+\mathrm{AlCl}_{3}$-treated; $\mathrm{FA}+\mathrm{AlCl}_{3}$, Ferulic acid $+\mathrm{AlCl}_{3}$-treated; $\mathrm{EA}+\mathrm{FA}+\mathrm{AlCl}_{3}$, Ellagic acid + Ferulic acid $+\mathrm{AlCl}_{3}$-treated; $\mathrm{EA}+\mathrm{AlCl}_{3}+\gamma$, Ellagic acid + $\mathrm{AlCl}_{3}$-treated $+\gamma$-irradiated; $\mathrm{FA}+\mathrm{AlCl}_{3}+\gamma$, Ferulic acid $+\mathrm{AlCl}_{3}$-treated $+\gamma$-irradiated; $\mathrm{EA}+\mathrm{FA}+\mathrm{AlCl}_{3}+\gamma$, Ellagic acid + Ferulic acid $+\mathrm{AlCl}_{3}$-treated $+\gamma$-irradiated; N.S: non-significant $(P>0.05)$. Data are represented as mean \pm SEM $(n=8) . \%$ change 1 , compared to control group; $\%$ change 2 , compared to $\gamma$-irradiated group; $\%$ change 3 , compared to $\mathrm{AlCl}_{3}$-treated group; $\%$ change 4 , compared to $\mathrm{AlCl}_{3}$-treated $\gamma$-irradiated group.

increased activities of liver enzymes. In line with our observation, it has been previously demonstrated that chronic $\mathrm{Al}$ exposure induce hepatotoxicity manifested by elevated liver enzymes ${ }^{[32]}$. Extensive damage to liver tissue via free radicals-mediated lipid peroxidation can result in membrane disorganization and subsequently decreases the membrane fluidity ${ }^{[33]}$. Thus, the increased activities of serum liver enzymes in our study are suggestive of severe hepatic injury as a result of $\gamma$ irradiation andlor $\mathrm{AlCl}_{3}$-treatment. Treatment with EA and/or FA effectively resulted in significant decreases in the activities of serum liver enzymes, suggesting that the leakage of enzymes from the hepatocellular membrane is decreased by the hepatocytes membrane-stabilizing action of EA and FA.

In the present study, the elevation of serum TC, TG and LDL-C levels which was accompanied by a decrease in HDL-C level in $\gamma$-irradiated rats might be attributed to increased fat mobilization from adipose tissues as a result of radiation-induced cellular biomembrane injury ${ }^{[34]}$. This finding is consistent with a previous report ${ }^{[35]}$, suggesting that the hyperlipidemia which observed after irradiation might be explained by an alteration in hepatic lipid metabolism and serum lipoproteins. Similarly, 
Table 4: Effects of EA and/or FA administration on SOD activity and GSH content in liver tissue of $\mathrm{AlCl}_{3}$-treated and/or $\gamma$-irradiated rats.

\begin{tabular}{|c|c|c|}
\hline Animal groups & $\begin{array}{c}\text { GSH } \\
\text { (mg/g tissue) }\end{array}$ & $\begin{array}{c}\text { SOD } \\
\text { (U/g tissue) }\end{array}$ \\
\hline $\mathbf{C}$ & $17.74 \pm 0.14$ & $47.41 \pm 0.47$ \\
\hline $\begin{array}{c}\gamma \\
\% \text { change } 1, P \text {-value }<\end{array}$ & $\begin{array}{c}10.65 \pm 0.36 \\
-39.96,0.001\end{array}$ & $\begin{array}{l}22.09 \pm 0.80 \\
-53.4,0.001\end{array}$ \\
\hline $\begin{array}{c}\mathrm{AlCl}_{3} \\
\% \text { change } 1, P \text {-value }<\end{array}$ & $\begin{array}{c}12.04 \pm 0.4 \\
-32.13,0.001\end{array}$ & $\begin{array}{c}17.31 \pm 0.46 \\
-63.48,0.001\end{array}$ \\
\hline $\begin{array}{c}\mathrm{AlCl}_{3}+\gamma \\
\% \text { change } 1, P \text {-value }<\end{array}$ & $\begin{array}{c}6.66 \pm 0.19 \\
-62.45,0.001\end{array}$ & $\begin{array}{c}15.29 \pm 0.67 \\
-67.74,0.001\end{array}$ \\
\hline $\begin{array}{c}\text { EA+ } \gamma \\
\% \text { change } 1, P \text {-value }< \\
\% \text { change } 2, P \text {-value }<\end{array}$ & $\begin{array}{c}15.19 \pm 7 \\
-14.37,0.001 \\
42.62,0.001 \\
\end{array}$ & $\begin{array}{c}32.83 \pm 0.3 \\
-30.75,0.001 \\
48.61,0.001 \\
\end{array}$ \\
\hline $\begin{array}{c}\text { FA }+\gamma \\
\% \text { change } 1, P \text {-value }< \\
\% \text { change } 2, P \text {-value }<\end{array}$ & $\begin{array}{c}16 \pm 0.1 \\
-9.8,0.001 \\
50.23,0.001 \\
\end{array}$ & $\begin{array}{c}39.79 \pm 0.4 \\
-16.07,0.001 \\
80.12,0.001 \\
\end{array}$ \\
\hline $\begin{array}{c}\text { EA }+\mathrm{FA}+\gamma \\
\% \text { change } 1, P \text {-value }< \\
\% \text { change } 2, P \text {-value }<\end{array}$ & $\begin{array}{c}16.65 \pm 9.6 \\
-6.14,0.001 \\
56.33,0.001 \\
\end{array}$ & $\begin{array}{c}44.84 \pm 0.56 \\
-5.42,0.001 \\
102.98,0.001 \\
\end{array}$ \\
\hline $\begin{array}{c}\mathrm{EA}+\mathrm{AlCl}_{3} \\
\% \text { change } 1, P \text {-value }< \\
\% \text { change } 3, P \text {-value }<\end{array}$ & $\begin{array}{l}16.16 \pm 0.1 \\
-8.9,0.001 \\
34.21,0.001 \\
\end{array}$ & $\begin{array}{c}35.83 \pm 0.54 \\
-24.42,0.001 \\
106.99,0.001 \\
\end{array}$ \\
\hline $\begin{array}{c}\text { FA+AlCl} \\
\% \text { change } 1, P \text {-value }< \\
\% \text { change } 3, P \text {-value }<\end{array}$ & $\begin{array}{c}16.25 \pm 0.16 \\
-8.3,0.001 \\
34.96,0.001 \\
\end{array}$ & $\begin{array}{c}41.73 \pm 0.31 \\
-11.98,0.001 \\
141.07,0.001 \\
\end{array}$ \\
\hline $\begin{array}{c}\text { EA+FA+AlCl } \\
\% \text { change } 1, P \text {-value }< \\
\% \text { change } 3, P \text {-value }< \\
\end{array}$ & $\begin{array}{l}16.97 \pm 6.1 \\
-4.34,0.01 \\
40.94,0.001 \\
\end{array}$ & $\begin{array}{c}46.15 \pm 0.37 \\
-2.65, \text { N.S } \\
166.6,0.001 \\
\end{array}$ \\
\hline $\begin{array}{c}\text { EA+AlCl }+\gamma \\
\% \text { change } 1, P \text {-value }< \\
\% \text { change } 4, P \text {-value }<\end{array}$ & $\begin{array}{c}14.37 \pm 0.19 \\
-18.99,0.001 \\
115.76,0.001 \\
\end{array}$ & $\begin{array}{c}25.71 \pm 0.38 \\
-45.77,0.001 \\
68.14,0.001 \\
\end{array}$ \\
\hline $\begin{array}{c}\text { FA+AlCl}+\gamma \\
\% \text { change } 1, P \text {-value }< \\
\% \text { change } 4, P \text {-value }<\end{array}$ & $\begin{array}{c}14.68 \pm 0.17 \\
-17.24,0.001 \\
120.42,0.001 \\
\end{array}$ & $\begin{array}{c}28.03 \pm 0.39 \\
-40.87,0.001 \\
83.32,0.001 \\
\end{array}$ \\
\hline $\begin{array}{c}\text { EA+FA+AlCl }+\gamma \\
\% \text { change } 1, P \text {-value }< \\
\% \text { change } 4, P \text {-value }<\end{array}$ & $\begin{array}{c}15.93 \pm 0.1 \\
-10.2,0.001 \\
139.18,0.001\end{array}$ & $\begin{array}{c}32.5 \pm 0.28 \\
-31.44,0.001 \\
112.55,0.001\end{array}$ \\
\hline
\end{tabular}

$\mathrm{GSH}$, reduced glutathione; SOD, superoxide dismutase; C, Control; $\gamma, \gamma$-irradiated; $\mathrm{AlCl}_{3}, \mathrm{AlCl}_{3}$-treated; $\mathrm{AlCl}_{3}+\gamma, \mathrm{AlCl}_{3}$-treated $+\gamma$-irradiated; EA $+\gamma$, Ellagic acid $+\gamma$-irradiated; FA $+\gamma$, Ferulic acid $+\gamma$-irradiated; EA $+\mathrm{FA}+\gamma$, Ellagic acid + Ferulic acid $+\gamma$-irradiated; EA $+\mathrm{AlCl}_{3}$, Ellagic acid $+\mathrm{AlCl}_{3}$-treated; $\mathrm{FA}+\mathrm{AlCl}_{3}$, Ferulic acid $+\mathrm{AlCl}_{3}$-treated; $\mathrm{EA}+\mathrm{FA}+\mathrm{AlCl}_{3}$, Ellagic acid + Ferulic acid $+\mathrm{AlCl}_{3}$-treated; $\mathrm{EA}+\mathrm{AlCl}_{3}+\gamma$, Ellagic acid + $\mathrm{AlCl}_{3}$-treated $+\gamma$-irradiated; $\mathrm{FA}+\mathrm{AlCl}_{3}+\gamma$, Ferulic acid $+\mathrm{AlCl}_{3}$-treated $+\gamma$-irradiated; $\mathrm{EA}+\mathrm{FA}+\mathrm{AlCl}_{3}+\gamma$, Ellagic acid + Ferulic acid $+\mathrm{AlCl}_{3}$-treated $+\gamma$-irradiated; N.S: non-significant $(P>0.05)$. Data are represented as mean \pm SEM $(n=8) . \%$ change 1 , compared to control group; $\%$ change 2 , compared to $\gamma$-irradiated group; $\%$ change 3 , compared to $\mathrm{AlCl}_{3}$-treated group; $\%$ change 4 , compared to $\mathrm{AlCl}_{3}$-treated $+\gamma$-irradiated group.

increased serum TC, TG and LDL-C levels with concomitant reduction of $\mathrm{HDL}-\mathrm{C}$ level in $\mathrm{AlCl}_{3}$-treated rats might be mediated by a direct effect on lipoprotein metabolism. In line with our findings, it has been previously demonstrated that Al-induced lipid peroxidation could arise from alteration of lipid metabolism and that is probably related to altered lipoprotein metabolism rather than a direct effect of cholesterol oxidation ${ }^{[36]}$. Administration of EA and/or FA alleviated $\mathrm{AlCl}_{3}$ and/or $\gamma$-radiation-induced dyslipidemia by reducing serum TC, TG and LDL-C levels, while increasing HDL-C levels. These findings are in agreement with previous reports showing hypolipidemic effects of EA and FA treatment ${ }^{[37,38]}$. It can be postulated that EA and FA can act in several ways to decrease serum TC, TG and LDL-C levels. First, uptake of TC and TG in the gastrointestinal tract could be inhibited; second, LDL-C could be eliminated from the blood via the LDL receptors; and finally, the activity of cholesterol-degrading enzymes could be increased.

MDA, a metabolic product of lipid peroxidation, is considered a biomarker for oxidative stress and cellular damage [39]. In addition, the formation of carbonyl derivatives of proteins is suggested to be a useful 
Table 5: Effects of EA and/or FA administration on iron and zinc concentrations in liver tissue of $\mathrm{AlCl}_{3}$-treated and/or $\gamma$-irradiated rats.

\begin{tabular}{|c|c|c|}
\hline Animal groups & $\begin{array}{c}\text { Fe } \\
(\mu \mathrm{g} / \mathrm{g} \text { tissue })\end{array}$ & $\begin{array}{c}\text { Zn } \\
(\mu \mathrm{g} / \mathrm{g} \text { tissue })\end{array}$ \\
\hline $\mathbf{C}$ & $78.98 \pm 1.0$ & $14.93 \pm 1.4$ \\
\hline $\begin{array}{c}\gamma \\
\% \text { change } 1, P \text {-value }<\end{array}$ & $\begin{array}{c}135.61 \pm 0.62 \\
71.7,0.001\end{array}$ & $\begin{array}{c}29.05 \pm 0.5 \\
94.57,0.001\end{array}$ \\
\hline $\begin{array}{c}\mathrm{AlCl}_{3} \\
\% \text { change } 1, P \text {-value }<\end{array}$ & $\begin{array}{l}99.05 \pm 3.38 \\
25.41,0.001 \\
\end{array}$ & $\begin{array}{l}26.16 \pm 0.30 \\
75.21,0.001\end{array}$ \\
\hline $\begin{array}{c}\mathrm{AlCl}_{3}+\gamma \\
\% \text { change } 1, P \text {-value }<\end{array}$ & $\begin{array}{c}161.91 \pm 2.82 \\
105,0.001\end{array}$ & $\begin{array}{c}32.79 \pm 0.91 \\
119.62,0.001 \\
\end{array}$ \\
\hline $\begin{array}{c}\text { EA }+\gamma \\
\% \text { change } 1, P \text {-value }< \\
\% \text { change } 2, P \text {-value }<\end{array}$ & $\begin{array}{c}119 \pm 0.38 \\
50.67,0.001 \\
-12.24,0.001 \\
\end{array}$ & $\begin{array}{c}18 \pm 0.18 \\
20.56,0.001 \\
-38.03,0.001 \\
\end{array}$ \\
\hline $\begin{array}{c}\text { FA }+\gamma \\
\% \text { change } 1, P \text {-value }< \\
\% \text { change } 2, P \text {-value }<\end{array}$ & $\begin{array}{c}114.51 \pm 1.3 \\
44.98,0.001 \\
-15.55,0.001 \\
\end{array}$ & $\begin{array}{c}19.28 \pm 0.21 \\
29.13,0.001 \\
-33.63,0.001 \\
\end{array}$ \\
\hline $\begin{array}{c}\mathrm{EA}+\mathrm{FA}+\gamma \\
\% \text { change } 1, P \text {-value }< \\
\% \text { change } 2, P \text {-value }<\end{array}$ & $\begin{array}{c}86.68 \pm 1.2 \\
9.74,0.001 \\
-36.08,0.001 \\
\end{array}$ & $\begin{array}{c}16.38 \pm 0.23 \\
9.71, \mathrm{~N} . \mathrm{S} \\
-43.61,0.001 \\
\end{array}$ \\
\hline $\begin{array}{c}\mathrm{EA}+\mathrm{AlCl}_{3} \\
\% \text { change } 1, P \text {-value }< \\
\% \text { change } 3, P \text {-value }<\end{array}$ & $\begin{array}{c}91.74 \pm 1.2 \\
16.15,0.001 \\
-7.38,0.001 \\
\end{array}$ & $\begin{array}{c}17.69 \pm 0.29 \\
18.48,0.01 \\
-32.37,0.001 \\
\end{array}$ \\
\hline $\begin{array}{c}\text { FA+AlCl } \mathrm{F}_{3} \\
\% \text { change } 1, P \text {-value }< \\
\% \text { change } 3, P \text {-value }<\end{array}$ & $\begin{array}{c}87.92 \pm 0.69 \\
11.31,0.001 \\
-11.23,0.001 \\
\end{array}$ & $\begin{array}{c}17.23 \pm 0.42 \\
15.4,0.05 \\
-34.13,0.001 \\
\end{array}$ \\
\hline $\begin{array}{c}\text { EA+FA+AlCl } \\
\% \text { change } 1, P \text {-value }< \\
\% \text { change } 3, P \text {-value }<\end{array}$ & $\begin{array}{c}80.12 \pm 0.53 \\
1.44, \text { N.S } \\
-19.11,0.001 \\
\end{array}$ & $\begin{array}{c}15.41 \pm 0.29 \\
3.21, \text { N.S } \\
-41.09,0.001\end{array}$ \\
\hline $\begin{array}{c}\mathrm{EA}+\mathrm{AlCl}_{3}+\gamma \\
\% \text { change } 1, P \text {-value }< \\
\% \text { change } 4, P \text {-value }<\end{array}$ & $\begin{array}{c}132.43 \pm 0.51 \\
67.67,0.001 \\
-18.2,0.001 \\
\end{array}$ & $\begin{array}{c}24.40 \pm 0.48 \\
63.42,0.001 \\
-25.58,0.001 \\
\end{array}$ \\
\hline $\begin{array}{c}\text { FA+AlCl }+\gamma \\
\% \text { change } 1, P \text {-value }< \\
\% \text { change } 4, P \text {-value }<\end{array}$ & $\begin{array}{c}130.48 \pm 0.55 \\
65.2,0.001 \\
-19.41,0.001 \\
\end{array}$ & $\begin{array}{c}22.95 \pm 0.99 \\
53.71,0.001 \\
-30,0.001 \\
\end{array}$ \\
\hline $\begin{array}{c}\text { EA+FA+AlCl}+\gamma \\
\% \text { change } 1, P \text {-value }< \\
\% \text { change } 4, P \text {-value }<\end{array}$ & $\begin{array}{c}94.09 \pm 1.86 \\
19.13,0.001 \\
-41.88,0.001 \\
\end{array}$ & $\begin{array}{c}18.81 \pm 0.32 \\
25.98,0.001 \\
-42.63,0.001 \\
\end{array}$ \\
\hline
\end{tabular}

C, Control; $\gamma, \gamma$-irradiated; $\mathrm{AlCl}_{3}, \mathrm{AlCl}_{3}$-treated; $\mathrm{AlCl}_{3}+\gamma, \mathrm{AlCl}_{3}$-treated $+\gamma$-irradiated; EA $+\gamma$, Ellagic acid $+\gamma$-irradiated; $\mathrm{FA}+\gamma$, Ferulic acid $+\gamma$ irradiated; EA $+\mathrm{FA}+\gamma$, Ellagic acid + Ferulic acid $+\gamma$-irradiated; $\mathrm{EA}+\mathrm{AlCl}_{3}$, Ellagic acid $+\mathrm{AlCl}_{3}$-treated; $\mathrm{FA}+\mathrm{AlCl}_{3}, \mathrm{Ferulic}$ acid $+\mathrm{AlCl}$-treated; $\mathrm{EA}+\mathrm{FA}+\mathrm{AlCl}_{3}$, Ellagic acid + Ferulic acid $+\mathrm{AlCl}_{3}$-treated; $\mathrm{EA}+\mathrm{AlCl}_{3}+\gamma$, Ellagic acid $+\mathrm{AlCl}_{3}$-treated $+\gamma$-irradiated; $\mathrm{FA}+\mathrm{AlCl}_{3}+\gamma, \mathrm{Ferulic}$ acid + $\mathrm{AlCl}_{3}$-treated $+\gamma$-irradiated; EA+FA+ $\mathrm{AlCl}_{3}+\gamma$, Ellagic acid + Ferulic acid $+\mathrm{AlCl}_{3}$-treated $+\gamma$-irradiated; N.S: non-significant $(P>0.05)$. Data are represented as mean $\pm \operatorname{SEM}(\mathrm{n}=8)$. \% change 1 , compared to control group; \% change 2 , compared to $\gamma$-irradiated group; \% change 3, compared to $\mathrm{AlCl}_{3}$-treated group; \% change 4, compared to $\mathrm{AlCl}_{3}$-treated $+\gamma$-irradiated group.

measure of oxidative damage to proteins ${ }^{[40]}$. Our results support the above observations by showing that $\mathrm{AlCl}_{3}$ treated and/or $\gamma$-irradiated rats had significantly increased levels of MDA and PCC, which might be reasonably attributed to the susceptibility of cellular lipids and proteins to free radicals attack. It has been demonstrated that ionizing radiation induces the peroxidation of lipids, which leads to structural and functional damage to cellular membranous molecules either directly by transferring energy or indirectly by the generation of oxygen-derived free hydroxyl radicals, superoxide and nitric oxide, which are the predominant cellular free radicals ${ }^{[41]}$. Likewise, it has been suggested that the toxic effects associated with $\mathrm{Al}$ are due to the generation of ROS, which results in the oxidative deterioration of cellular lipids. The mechanism of Al pro-oxidant action may occur through its interaction with the membranes and subtle changes in the rearrangement of lipids which could attack and facilitate the propagation of lipid peroxidation ${ }^{[42]}$. On the other hand, the carbonyl derivatives of proteins may result from oxidative modification of amino acid side chains and reactive oxygen-mediated peptide cleavage, leading to changes in protein function, chemical fragmentation or 
increased susceptibility to a proteolytic attack ${ }^{[43]}$ SOD is considered the first line of defense against the deleterious effects of ROS. It catalyzes the rapid removal of superoxide radicals, generating $\mathrm{H}_{2} \mathrm{O}_{2}$. The inhibition of SOD activity may result in an increased flux of superoxide in cellular compartments which may be the reason for the increased lipid peroxidative indices. GSH is an antioxidant and a powerful nucleophile, critical for cellular protection such as detoxification of ROS, conjugation and excretion of toxic molecules and control of inflammatory cytokine cascade. Depletion of GSH in tissues leads to impairment of the cellular defense against ROS, and may result in peroxidative injury. In the present study, the observed decrease in SOD activity in $\gamma$-irradiated rats could be explained by feedback inhibition or oxidative inactivation of SOD due to excess ROS generation. Likewise, $\gamma$-irradiated rats showed depletion in GSH content.

Noteworthy, depletion of GSH in vitro and in vivo is

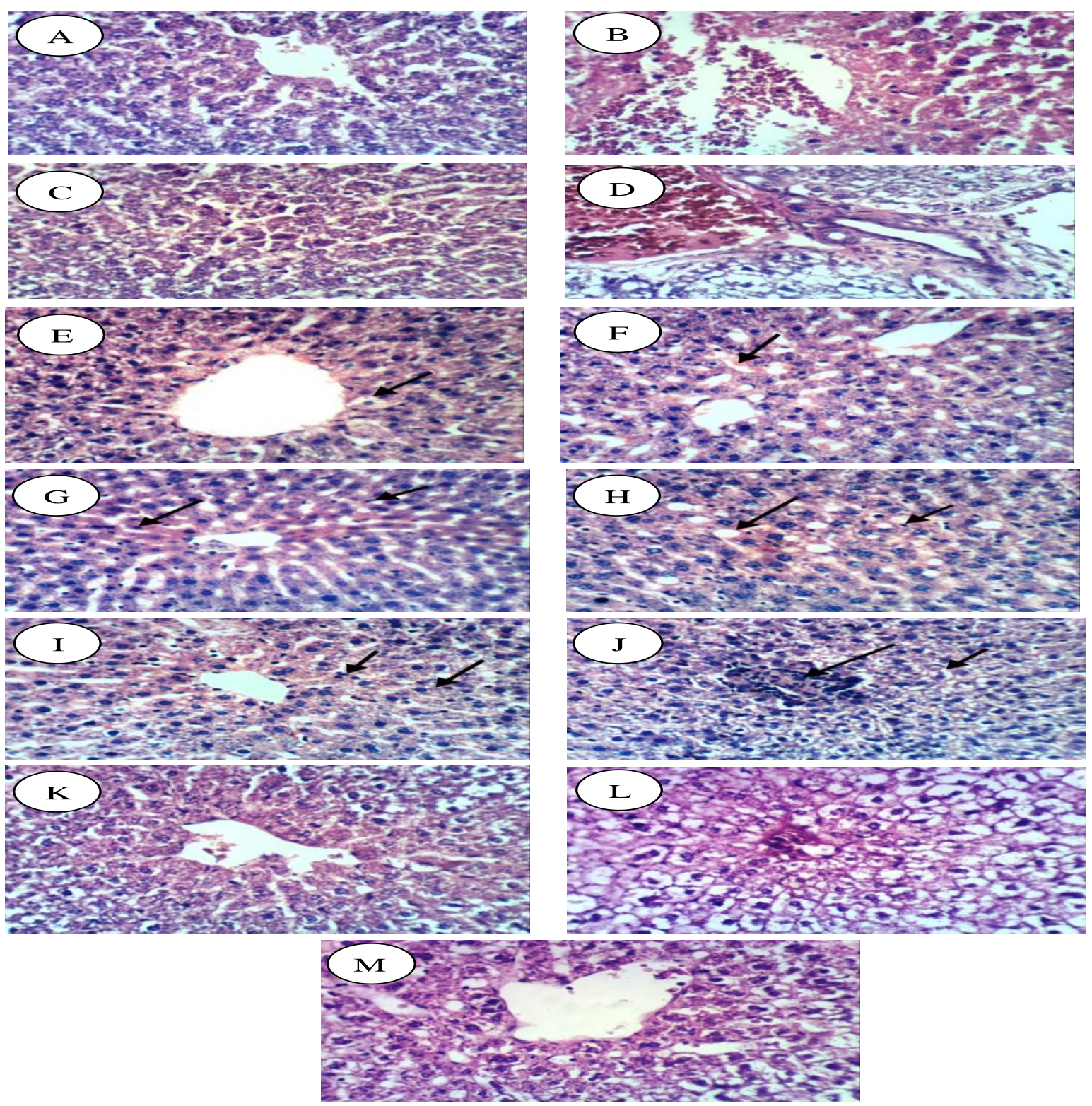

Fig. 1: Histological examination of hepatic sections stained with hematoxylin and eosin. (A) Control group showing normal hepatic architecture. (B) $\gamma$-irradiated group showing focal hepatic haemorrhage. (C) $\mathrm{AlCl}_{3}$-treated group showing cytoplasmic vacuolization of hepatocytes. (D) $\mathrm{AlCl}_{3}+\gamma$ group showing cytoplasmic vacuolization of hepatocytes, congestion of hepatoportal blood vessel and deposition of collagen fibers in portal tract. (E) EA $+\gamma$ group showing slight hydropic degeneration of centrolobular hepatocytes. (F) $\mathrm{FA}+\gamma$ group showing slight dilatation of hepatic sinusoids. (G) EA+FA+ $\gamma$ group showing slight Kupffer cells activation and necrosis of sporadic hepatocytes. (H) EA+ $\mathrm{AlCl}_{3}$ group showing slight Kupffer cells activation and fatty change (cytoplasmic vacuolization) of focal hepatocytes. (I) $\mathrm{FA}+\mathrm{AlCl}_{3}$ group showing Kupffer cells activation and slight hydropic degeneration of hepatocytes. (J) EA+FA+ $\mathrm{AlCl}_{3}$ group showing cytoplasmic vacuolization of hepatocytes and slight focal hepatic necrosis associated with inflammatory cells infiltration. (K) EA+ $\mathrm{AlCl}_{3}+\gamma$ group showing slight hydropic degeneration of some hepatocytes. (L) $\mathrm{FA}+\mathrm{AlCl}_{3}+\gamma$ group showing ballooning degeneration of hepatocytes. (M) $\mathrm{EA}+\mathrm{FA}+\mathrm{AlCl}{ }_{3}+\gamma$ group showing slight vacuolization of hepatocytes, ballooning degeneration of hepatocytes. Arrows indicate hepatocellular histological alterations. 
known to cause an inhibition of the glutathione peroxidase activity and has been shown to increase lipid peroxidation ${ }^{[44]}$. In our study, $\mathrm{AlCl}_{3}$-treated rats revealed a decrease in SOD activity as well as a reduction in GSH content. Interestingly, $\mathrm{Al}$ accumulation in hepatocytes was associated with increased membrane lipid peroxidation thus Al may alter the activity of a number of tissue antioxidant enzymes, such as SOD ${ }^{[45]}$. The observed decrease in the activity of $\mathrm{SOD}$ in $\mathrm{AlCl}_{3}$-treated rats, compared to the control rats, could be the result of reduced synthesis of the enzyme due to higher intracellular concentrations of $\mathrm{Al}$ [46]. Also, it has been reported that, at high doses, $\mathrm{Al}$ might affect GSH synthesis by decreasing glutathionesynthase activity, which would result in reduced GSH levels ${ }^{[47]}$.

Our results showed that the administration of EA and/or FA to $\mathrm{AlCl}_{3}$-treated and/or $\gamma$-irradiated rats significantly decreased the levels of MDA and PCC, while increasing SOD activity and GSH content. These actions might be attributed to the effective antioxidant properties of EA and FA. It has been reported that phenolic compounds can act by scavenging free radicals. EA ${ }^{[48]}$ and FA ${ }^{[49]}$, being phenolic compounds, quench free radicals, inhibit lipid peroxidation, diminish the production of protein carbonyl groups, enhance the SOD and GSH-dependent protection and ultimately decrease the burden to antioxidants, thereby enhancing the antioxidant status.

Trace elements such as iron and zinc play a vital role in maintaining the oxidative status of the cell. In the current study, $\gamma$-irradiation andlor $\mathrm{AlCl}_{3}$-treatment caused an increase in the liver tissue levels of Fe and Zn. It has been reported that exposure to gamma radiation [50] and $\mathrm{Al}{ }^{[51]}$ could promote disruptions in the trace elements balance. Although $\mathrm{Al}$ is not a transition metal, and therefore, cannot initiate peroxidation, many studies have searched for a correlation between $\mathrm{Al}$ accumulation and oxidative damage in tissues. An in vitro study indicated that $\mathrm{Al}$ greatly accelerates iron-mediated lipid peroxidation ${ }^{[52]}$. Interestingly, exposure to $\mathrm{Al}$ resulted in $\mathrm{Al}$ ions replacing iron, which would then lead to a reduction in $\mathrm{Fe}^{2+}$ binding to ferritin. Free iron ions released from biological complexes by Al can catalyze hydroperoxides decomposition to hydroxyl radicals via Fenton's reaction. This high hydroxyl radical reactivity could initiate the peroxidation of membrane lipids, causing membrane damage. In the present study, administration of EA and/or FA positively modulated the levels of iron and zinc. This is mainly because of the remarkable antioxidant action of EA and FA. Notably, EA plays a protective role upon major and trace elements against $\mathrm{AlCl}_{3}$-induced stress conditions ${ }^{[53]}$. Despite the direct scavenging of ROS, FA can chelate the ferrous ion and decrease the formation of hydroxyl radical via inhibition of iron-dependent Fenton's reaction ${ }^{[54]}$.

Structure-activity relationship studies have demonstrated that the two lactone groups of EA can act as a hydrogen bond donor and acceptor, which might be involved in the free radical scavenging action and decreased free radicals-mediated lipid peroxidation ${ }^{[55]}$. On the other hand, FA possesses distinct structural motifs that can possibly contribute to the antioxidant property of this compound. The presence of electron donating groups on the benzene ring [3-methoxy and more importantly 4hydroxyl] of FA gives additional property for terminating free radical chain reaction. The next functionality - the carboxylic acid group in FA with adjacent unsaturated $\mathrm{C}-\mathrm{C}$ double bond can provide additional attack sites for free radicals and thus prevent them from attacking the membrane. In addition, the carboxylic acid group also acts as an anchor of FA by which it binds to the lipid bilayer providing some protection against lipid peroxidation ${ }^{[56]}$.

Histological examination of the liver tissues strongly supports the hepatoprotective effect of EA and FA, where the $\mathrm{AlCl}_{3}$ and/or $\gamma$-radiation-induced hepatic histological alterations were attenuated upon administration of EA and/or FA. Previously, it has been demonstrated that EA treatment reduced alcohol-induced hepatic pathological changes [57]. Furthermore, carbon tetrachloride-induced histological changes in the liver were significantly attenuated by FA treatment ${ }^{[58]}$.

\section{Conclusions}

In conclusion, our results indicate that EA and FA could attenuate $\gamma$ radiation and/or $\mathrm{AlCl}_{3}$-induced oxidative damage via ROS scavenging-mediated mechanisms, thereby modulating lipids and proteins oxidation, increasing antioxidant enzyme activities and improving endogenous antioxidant status. Our findings provide new perspectives on the therapeutic use of EA and FA in alleviating the oxidative damage induced by $\gamma$ radiation and/or $\mathrm{AlCl}_{3}$. Further studies in human populations are warranted to support our observations of the antioxidant potential of EA and FA.

\section{References}

1) Kohen, R. and Nyska, A. (2002). Oxidation of biological systems: oxidative stress phenomena, antioxidants, redox reactions, and methods for their quantification. Toxicol. Pathol. 30(6):620-650.

2) Spitz, D. R., Azzam, E. I., Li, J. J. and Gius, D. (2004). Metabolic oxidation/reduction reactions and cellular responses to ionizing radiation: a unifying concept in stress response biology. Cancer Metastasis Rev. 23(3-4):311-322.

3) Lione, A. (1985). Aluminum toxicology and the aluminum-containing medications. Pharmacol. Ther. 29(2):255-285.

4) Klöppel, H., Fliedner, A. and Kördel, W. (1997). Behavior and ecotoxicology of aluminium in soil and water-review of the scientific literature. Chemosphere 35(1-2):353-363.

5) Fatima, S. K., Prabhavathi, P. A., Padmavathi, P. and Reddy, P. P. (2001). Analysis of chromosomal aberrations in men occupationally exposed to cement dust. Mutat. Res. 490(2):179-186.

6) Yokel, R. A. and McNamara, P. J. (2001). Aluminium toxicokinetics: an updated minireview. 
Pharmacol. Toxicol. 88(4):159-167.

7) Becaria, A., Campbell, A. and Bondy, S. C. (2002). Aluminium as a toxicant. Toxicol. Ind. Health. 18(7):309-320.

8) Duthie, G. G., Duthie, S. J. and Kyle, J. A. (2000). Plant polyphenols in cancer and heart disease: implications as nutritional antioxidants. Nutr. Res. Rev. 13(1):79-106.

9) Vattem, D. A. and Shetty, K. (2005). Biological functionality of ellagic acid: A review. J. Food Biochem. 29(3):234-266.

10) Seeram, N. P., Adams, L. S., Henning, S. M., Niu, Y., Zhang, Y., Nair, M. G. and Heber, D. (2005). In vitro antiproliferative, apoptotic and antioxidant activities of punicalagin, ellagic acid and a total pomegranate tannin extract are enhanced in combination with other polyphenols as found in pomegranate juice. J. Nutr. Biochem. 16(6):360367.

11) Srinivasan, M., Sudheer, A. R. and Menon, V. P. (2007). Ferulic Acid: therapeutic potential through its antioxidant property. J. Clin. Biochem. Nutr. 40(2):92-100.

12) Murakami, A., Nakamura, Y., Koshimizu, K., Takahashi, D., Matsumoto, K., Hagihara K., Taniguchi, H., Nomura, E., Hosoda, A., Tsuno, T., Maruta, Y., Kim, H. W., Kawabata, K. and Ohigashi, H. (2002). FA15, a hydrophobic derivative of ferulic acid, suppresses inflammatory responses and skin tumor promotion: comparison with ferulic acid. Cancer Lett. 180(2):121-129.

13) Koc, M., Taysi, S., Buyukokuroglu, M. E. and Bakan, N. (2003). Melatonin protects rat liver against irradiation-induced oxidative injury. $J$. Radiat. Res. 44(3):211-215.

14) Krasovskiŭ, G. N., Vasukovich, L. Y. and Chariev, O. G. (1979). Experimental study of biological effects of leads and Aluminium following oral administration. Environ. Health Perspect. 30: 47-51.

15) Lowry, O. H., Rosebrough, N. J., Farr, A. L. and Randall, R. J. (1951). Protein measurement with the Folin phenol reagent. J. Biol. Chem. 193(1): 265-275.

16) Bergmeyer, H. U., Scheibe, P. and Wahlefeld, A. W. (1978). Optimization of methods for aspartate aminotransferase and alanine aminotransferase. Clin. Chem. 24(1): 58-73.

17) Empfehlungen der Deutschen Gesellschaft für Klinische Chemie (1972). Standard-Methode zur Bestimmung der Aktivität der alkalischen phosphatase. Z. Klin. Chem. U. Klin. Biochem. 10: 191.

18) Persijn, J. P. and van der Slik, W. (1976). A new method for the determination of gammaglutamyltransferase in serum. J. Clin. Chem. Clin. Biochem. 14(9):421-427.

19) Meiattini, F., Prencipe, L., Bardelli, F., Giannini, G. and Tarli, P. (1978). The 4-hydroxybenzoate/4- aminophenazone chromogenic system used in the enzymic determination of serum cholesterol. Clin. Chem. 24(12):2161-2165.

20) Grove, T. H. (1979). Effect of reagent $\mathrm{pH}$ on determination of high-density lipoprotein cholesterol by precipitation with sodium phosphotungstatemagnesium. Clin. Chem. 25(4):560-564.

21) Bucolo, G. and David, H. (1973). Quantitative determination of serum triglycerides by the use of enzymes. Clin. Chem. 19(5):476-482.

22) Friedewald, W. T., Levy, R. I. and Fredrickson, D. S. (1972). Estimation of the concentration of lowdensity lipoprotein cholesterol in plasma, without use of the preparative ultracentrifuge. Clin. Chem. 18(6):499-502.

23) Placer, Z. A., Cushman, L. L. and Johnson, B. C. (1966). Estimation of product of lipid peroxidation (malonyldialdehyde) in biochemical systems. Anal. Biochem. 16(2):359-364.

24) Reznick, A. Z. and Packer, L. (1994). Oxidative damage to proteins: spectrophotometric method for carbonyl assay. Methods Enzymol. 233:357-363.

25) Tietze, F. (1969). Enzymic method for quantitative determination of nanogram amounts of total and oxidized glutathione: applications to mammalian blood and other tissues. Anal Biochem. 27(3):502522.

26) Sun, Y., Oberley, L. W. and Li, Y. (1988). A simple method for clinical assay of superoxide dismutase. Clin. Chem. 34(3):497-500.

27) Watanabe, M. (1996). Flameless atomic absorption spectrometry for analysis of trace elements in biological material. Nippon Rinsho-Japanese J. Clin. Med. 54(1):228-232.

28) Riley, P. A. (1994). Free radicals in biology: oxidative stress and the effects of ionizing radiation. Int. J. Radiat. Biol. 65(1):27-33.

29) Barabasz, W., Albinska, D., Jaskowska, M. and Lipiec, J. (2002). Ecotoxicology of aluminium. Pol. J. Environ. Stud. 11(3):199-203.

30) Sargazi, M., Shenkin, A. and Roberts, N. B. (2006). Aluminium-induced injury to kidney proximal tubular cells: Effects on markers of oxidative damage. J. Trace Elem. Med. Biol. 19(4):267-273.

31) Adaramoye, O., Ogungbenro, B., Anyaegbu, 0. and Fafunso, M. (2008). Protective effects of extracts of Vernonia amygdalina, Hibiscus sabdariffa and vitamin $\mathrm{C}$ against radiation-induced liver damage in rats. J. Radiat. Res. 49(2):123-131.

32) Bouasla, I., Bouasla, A., Boumendjel, A., El Feki, A. and Messarah, M. (2014). Antioxidant effect of alpha lipoic acid on hepatotoxicity induced by aluminium chloride in rats. Int. J. Pharm. Sci. Rev. Res. 29(2):19-25.

33) Recknagel, R. O. and Ghoshal, A. K. (1966). Quantitative estimation of peroxidative degeneration of rat liver microsomal and mitochondrial lipids after carbon tetrachloride poisoning. Exp. Mol. Pathol. 5(5):413-426. 
34) Onody, A., Csonka, C., Giricz, Z. and Ferdinandy, P. (2003). Hyperlipidemia induced by a cholesterol-rich diet leads to enhanced peroxynitrite formation in rat hearts. Cardiovasc. Res. 58(3):663-670.

35) Azab, K. S., Bashandy, M., Salem, M., Ahmed, O., Tawfik, Z. and Helal, H. (2011). Royal jelly modulates oxidative stress and tissue injury in gamma irradiated male wister albino rats. $N$. Am. J. Med. Sci. 3(6):268-276.

36) Belaïd-Nouira, Y., Bakhta, H., Bouaziz, M., Flehi-Slim, I., Haouas, Z. and Ben Cheikh, H. (2012). Study of lipid profile and parieto-temporal lipid peroxidation in $\mathrm{AlCl}_{3}$ mediated neurotoxicity. Modulatory effect of fenugreek seeds. Lipids Health Dis. 11:16.

37) Park, S. H., Kim, J. L, Lee, E. S., Han, S. Y., Gong, J. H., Kang, M. K. and Kang, Y. H. (2011). Dietary ellagic acid attenuates oxidized LDL uptake and stimulates cholesterol efflux in murine macrophages. J. Nutr. 141(11):1931-1937.

38) Wilson, T. A., Nicolosi, R. J., Woolfrey, B. and Kritchevsky, D. (2007). Rice bran oil and oryzanol reduce plasma lipid and lipoprotein cholesterol concentrations and aortic cholesterol ester accumulation to a greater extent than ferulic acid in hypercholesterolemic hamsters. J. Nutr. Biochem. 18(2):105-112.

39) Nordberg, J. and Arnér, E. S. (2001). Reactive oxygen species, antioxidants and the mammalian thioredoxin system. Free Radic. Biol. Med. 31(11):1287-1317.

40) Sundari, P. N., Wilfred, G. and Ramakrishna, B. (1997). Does oxidative protein damage play a role in the pathogenesis of carbon tetrachloride-induced liver injury in the rat? Biochim. Biophys. Acta 1362(2-3):169-176.

41) Joshi, R., Kamat, J. P. and Mukherjee, T. (2007). Free radical scavenging reactions antioxidant activity of embelin: biochemical and pulse radiolytic studies. Chem. Biol. Interact. 167(2):125-134.

42) Anane, R. and Creppy, E. E. (2001). Lipid peroxidation as a pathway to aluminium cytotoxicity in human skin fibroblast cultures: prevention by superoxide dismutase+catalase and vitamins $\mathrm{E}$ and C. Hum. Exp. Toxicol. 20(9):477-481.

43) Dröge, W. (2002). Free radicals in the physiological control of cell function. Physiol. Rev. 82(1):47-95.

44) Jagetia, G. C. and Reddy, T. K. (2005). Modulation of radiation-induced alteration in the antioxidant status of mice by naringin. Life Sci. 77(7):780-794.

45) Moumen, R., Ait-Oukhatar, N., Bureau, F., Fleury, C., Bouglé, D., Arhan, P., Neuville, D. and Viader, F. (2001). Aluminium increases xanthine oxidase activity and disturbs antioxidant status in the rat. J. Trace Elem. Med. Biol. 15(2-3):89-93.

46) Nehru, B. and Anand, P. (2005). Oxidative damage following chronic aluminium exposure in adult and pup rat brains. J. Trace Elem. Med. Biol. 19(23):203-208.

47) Orihuela, D., Meichtry, V., Pregi, N. and Pizarro, M. (2005). Short-term oral exposure to aluminium decrease glutathione intestinal levels and changes enzyme activities involved in its metabolism. $J$. Inorg. Biochem. 99(9):1871-1878.

48) Priyadarsini, K. I., Khopde, S. M., Kumar, S. S. and Mohan, H. (2002). Free radical studies of ellagic acid, a natural phenolic antioxidant. J. Agric. Food Chem. 50(7):2200-2206.

49) Kikuzaki, H., Hisamoto, M., Hirose, K., Akiyama, K. and Taniguchi, H. (2002). Antioxidant properties of ferulic acid and its related compounds. J. Agric. Food Chem. 50(7):2161-2168.

50) Elnimr, T. and Abdel-Rahim, S. M. (1998). Effect of gamma-radiation on some elements in certain organs of albino rats. Biol. Trace Elem. Res. 62(12):25-30.

51) Fiejka, M., Długaszek, M., Graczyk, A., Slowikowska, M. and Górska, P. (2001). Effects of aluminium and deferoxamine on concentration of essential elements in animals experimentally intoxicated with aluminium. Med. Dosw. Mikrobiol. 53(1):101-106.

52) Ward, R. J., Zhang, Y. and Crichton, R. R. (2001). Aluminium toxicity and iron homeostasis. J. Inorg. Biochem. 87(1-2):9-14.

53) Özkaya, A., Çiftçi, H., Dayangaç, A., Çevrimli, B. S., Ölçücü, A. and Çelik, S. (2013). Effects of ellagic acid and hesperetin on levels of some elements in livers of aluminium-induced rats. Turk. $J$. Bioch. 38(3):345-349.

54) Zhang, Z., Wei, T., Hou, J., Li, G., Yu, S. and Xin, W. (2003). Ion-induced damage and apoptosis in cerebellar granule cells: attenuation by tetramethylpyrazine and ferulic acid. Eur. J. Pharmacol. 467(1-3):41-47.

55) Kilic, I., Yeşiloğlu, Y. and Bayrak, Y. (2014). Spectroscopic studies on the antioxidant activity of ellagic acid. Spectrochim. Acta A. Mol. Biomol. Spectrosc. 130:447-452.

56) Kanski, J., Aksenova, M., Stoyanova, A. and Butterfield, D. A. (2002). Ferulic acid antioxidant protection against hydroxyl and peroxyl radical oxidation in synaptosomal and neuronal cell culture systems in vitro: structure-activity studies. J. Nutr. Biochem. 13(5):273-281.

57) Devipriya, N., Sudheer, A. R., Vishwanathan, $P$. and Menon, V. P. (2008). Modulatory potential of ellagic acid, a natural plant polyphenol on altered lipid profile and lipid peroxidation status during alcohol-induced toxicity: a pathohistological study. $J$. Biochem. Mol. Toxicol. 22(2):101-112.

58) Kim, H. Y., Park, J., Lee, K. H., Lee, D. U., Kwak, J. H., Kim, Y. S. and Lee, S. M. (2011). Ferulic acid protects against carbon tetrachloride-induced liver injury in mice. Toxicology 282(3):104-111. 